\title{
Algebro-Geometric Solutions of the Coupled Chaffee-Infante Reaction Diffusion Hierarchy
}

\author{
Chao Yue $\mathbb{D i}^{1}$ and Tiecheng Xia ${ }^{2}$ \\ ${ }^{1}$ College of Medical Information Engineering, Shandong First Medical University \& Shandong Academy of Medical Sciences, \\ Taian 271000, China \\ ${ }^{2}$ Department of Mathematics, Shanghai University, Shanghai 200444, China
}

Correspondence should be addressed to Chao Yue; yuechao_71@163.com

Received 17 October 2020; Accepted 18 May 2021; Published 22 July 2021

Academic Editor: Wen-Xiu Ma

Copyright (C) 2021 Chao Yue and Tiecheng Xia. This is an open access article distributed under the Creative Commons Attribution License, which permits unrestricted use, distribution, and reproduction in any medium, provided the original work is properly cited.

\begin{abstract}
The coupled Chaffee-Infante reaction diffusion (CCIRD) hierarchy associated with a $3 \times 3$ matrix spectral problem is derived by using two sets of the Lenard recursion gradients. Based on the characteristic polynomial of the Lax matrix for the CCIRD hierarchy, we introduce a trigonal curve $\mathscr{K}_{m-2}$ of arithmetic genus $m-2$, from which the corresponding Baker-Akhiezer function and meromorphic functions on $\mathscr{K}_{m-2}$ are constructed. Then, the CCIRD equations are decomposed into Dubrovintype ordinary differential equations. Furthermore, the theory of the trigonal curve and the properties of the three kinds of Abel differentials are applied to obtain the explicit theta function representations of the Baker-Akhiezer function and the meromorphic functions. In particular, algebro-geometric solutions for the entire CCIRD hierarchy are obtained.
\end{abstract}

\section{Introduction}

It is significantly important to search for solutions of nonlinear partial differential equations of mathematical physics. There are many methods to find the exact solutions $[1,2]$ and approximate solutions [1-3] of various nonlinear partial differential equations. Reaction diffusion equations are effective and important mathematical models, which contribute to explaining processes of the transition, diffusion, and fluidity of matter. Constructing exact solutions of such equations has been widely used in mathematics, physics, chemistry, biology, and other fields. Therefore, it is necessary for us to study algebro-geometric constructions of the coupled Chaffee-Infante reaction diffusion (CCIRD) hierarchy associated with a $3 \times 3$ matrix spectral problem. The third member in the hierarchy is

$$
\begin{gathered}
u_{1, t_{2}}=12 u_{1, x x}+24\left(u_{1}-u_{1}^{2} u_{2}\right), \\
u_{2, t_{2}}=-12 u_{2, x x}-24\left(u_{2}-u_{2}^{2} u_{1}\right),
\end{gathered}
$$

which is called the CCIRD equation compared with Equation (0.4) in Ref. [4].

Algebro-geometric solution is closely associated with the inverse spectral theory $[5,6]$, and the solution of the $\mathrm{KdV}$ equation with an initial value problem was solved by the use of the method in Ref. [7]. Over the recent decades, integrable equations related to $2 \times 2$ matrix spectral problems have been extensively researched. Several systematic methods have been developed to construct algebro-geometric solutions for integrable equations such as KdV, KadomtsevPetviashvili equation, modified KdV, sine-Gordon, Ablowitz-Kaup-Newell-Segur, the Camassa-Holm equations, and Ablowitz-Ladik lattice [8-27]. But the study of algebrogeometric solutions of the whole hierarchy of $3 \times 3$ is still a challenging problem. Fortunately, in Ref. [28], a unified framework was proposed to yield algebro-geometric solutions of the whole Boussinesq hierarchy. Based on the work of that, a systematic method was proposed to define the trigonal curve and develop the framework to analyse soliton equations associated with the $3 \times 3$ matrix spectral problems, from which the algebro-geometric solutions of some entire 
hierarchies are obtained [29-34]. In Ref. [29], algebrogeometric quasi-periodic solutions to the three-wave resonant interaction hierarchy related to the trigonal curve with three infinite points were obtained. Wang and Geng constructed algebro-geometric solutions of a new hierarchy of soliton equations associated with a $3 \times 3$ matrix spectral problem [30] based on the methods used in [28, 29]. Later, $\mathrm{Ma}$ analysed the four-component AKNS soliton hierarchy, particularly asymptotics of the Baker-Akhiezer functions, in such a way that it proposes a general theory applicable to soliton hierarchies associated with $3 \times 3$ matrix spectral problems [31]. As a continuous study of [31], Ma constructed algebro-geometric solutions of the four-component AKNS soliton hierarchy in terms of a general theory of trigonal curves [32]. However, as far as we know, algebro-geometric solutions to the CCIRD hierarchy have not been investigated. The most important result of this paper is to give the explicit algebro-geometric solutions to the CCIRD hierarchy related to $3 \times 3$ matrix spectral problems by using the approaches used in [28-30], which complements the existing works in this area.

The outline of this paper is as follows. In Section 2, we obtain the CCIRD hierarchy related to a $3 \times 3$ matrix spectral problem based on the Lenard recursion equations. In Section 3 , a trigonal curve $\mathscr{K}_{m-2}$ of arithmetic genus $m-2$ with three infinite points is introduced by the use of the characteristic polynomial of the Lax matrix for the stationary CCIRD equations, from which the stationary Baker-Akhiezer function and associated meromorphic functions are given on $\mathscr{K}_{m-2}$. Then, the stationary CCIRD equations are decomposed into the system of Dubrovin-type ordinary differential equations. In Section 4, we present the explicit theta function representations of the stationary Baker-Akhiezer function, of the meromorphic functions, and, in particular, of the potentials for the entire stationary CCIRD hierarchy. In Section 5, we extend all the Baker-Akhiezer functions, the meromorphic functions, the Dubrovin-type equations, and the theta function representations dealt with in Sections 3 and 4 to the time-dependent case.

\section{The CCIRD Hierarchy}

In the section, we shall derive the CCIRD hierarchy associated with a $3 \times 3$ spectral problem:

$$
\begin{gathered}
\psi_{x}=U \psi \\
U=\left(\begin{array}{ccc}
i \lambda & u_{1} & 1 \\
u_{2} & -i \lambda & 1 \\
1 & 1 & -2 i \lambda
\end{array}\right), \\
\psi=\left(\begin{array}{l}
\psi_{1} \\
\psi_{2} \\
\psi_{3}
\end{array}\right)
\end{gathered}
$$

where the potential $u=\left(u_{1}, u_{2}\right)^{T}$ and $\lambda$ is a spectral parameter. Next, we introduce the Lenard gradient sequences

$$
\begin{array}{ll}
K g_{j-1}=J g_{j}, & \left.g_{j}\right|_{u_{i}=0}=0, j \geq 0, \\
K \widehat{g}_{j-1}=J \widehat{g}_{j}, & \left.\widehat{g}_{j}\right|_{u_{i}=0}=0, j \geq 0,
\end{array}
$$

with two initial points

$$
\begin{aligned}
& g_{-1}=(0,0,0,0,0,0,1,0)^{T}, \\
& \widehat{\mathfrak{g}}_{-1}=(0,0,0,0,0,0,0,1)^{T},
\end{aligned}
$$

and two operators are defined as

$$
K=\left(\begin{array}{cccccccc}
\partial & 1 & 0 & 0 & 0 & -1 & -2 u_{1} & -u_{1} \\
1 & \partial & 0 & -u_{1} & 0 & 0 & -1 & -2 \\
0 & 0 & \partial & 1 & -1 & 0 & 2 u_{2} & u_{2} \\
0 & -u_{2} & 1 & \partial & 0 & 0 & 1 & -1 \\
0 & 0 & -1 & 0 & \partial & u_{2} & 1 & 2 \\
-1 & 0 & 0 & 0 & u_{1} & \partial & -1 & 1 \\
-u_{2} & 0 & u_{1} & 1 & 0 & -1 & \partial & 0 \\
0 & -1 & 0 & -1 & 1 & 1 & 0 & \partial \\
2 i & 0 & 0 & 0 & 0 & 0 & 0 & 0 \\
0 & -i & 0 & 0 & 0 & 0 & 0 & 0 \\
0 & 0 & -2 i & 0 & 0 & 0 & 0 & 0 \\
0 & 0 & 0 & -3 i & 0 & 0 & 0 & 0 \\
0 & 0 & 0 & 0 & i & 0 & 0 & 0 \\
0 & 0 & 0 & 0 & 0 & 3 i & 0 & 0 \\
-u_{2} & 0 & u_{1} & 1 & 0 & -1 & \partial & 0 \\
0 & -1 & 0 & -1 & 1 & 1 & 0 & \partial
\end{array}\right),
$$

Then, the sequences $\left\{g_{j}\right\}$ and $\left\{\widehat{g}_{j}\right\}, j \geq 0$, can be uniquely determined and the first several members read as

$$
\begin{aligned}
g_{0}= & \left(i u_{1},-i, i u_{2}, \frac{1}{3} i,-i, \frac{1}{3} i, 0,0\right)^{T}, \\
\widehat{g}_{0}= & \left(\frac{1}{2} i u_{1},-2 i, \frac{1}{2} i u_{2},-\frac{1}{3} i,-2 i,-\frac{1}{3} i, 0,0\right)^{T}, \\
g_{1}= & \left(\frac{1}{2} u_{1, x}-\frac{2}{3},-\frac{2}{3} u_{1},-\frac{1}{2} u_{2, x}-\frac{2}{3},-\frac{2}{3} u_{2},-\frac{2}{3} u_{2},\right. \\
& \left.-\frac{2}{3} u_{1}, \frac{1}{2} u_{1} u_{2}+1, \frac{8}{9}\right)^{T}, \\
\widehat{g}_{1}= & \left(\frac{1}{4} u_{1, x}+\frac{2}{3}, \frac{2}{3} u_{1},-\frac{1}{4} u_{2, x}+\frac{2}{3}, \frac{2}{3} u_{2}, \frac{2}{3} u_{2},\right. \\
& \left.\frac{2}{3} u_{1}, \frac{1}{4} u_{1} u_{2}-1, \frac{19}{9}\right)^{T} .
\end{aligned}
$$


In order to obtain the CCIRD hierarchy, we solve the stationary zero-curvature equation

$$
V_{x}-[U, V]=0, \quad V=\left(V_{i j}\right)_{3 \times 3},
$$

which is equivalent to

$$
\begin{gathered}
V_{11, x}+u_{2} V_{12}+V_{13}-u_{1} V_{21}-V_{31}=0, \\
V_{22, x}-u_{2} V_{12}+u_{1} V_{21}+V_{23}-V_{32}=0, \\
V_{33, x}-V_{13}-V_{23}+V_{31}+V_{32}=0, \\
V_{12, x}-2 i \lambda V_{12}+u_{1}\left(V_{11}-V_{22}\right)+V_{13}-V_{32}=0, \\
V_{13, x}+i \lambda V_{13}+V_{11}-V_{33}+V_{12}-u_{1} V_{23}=0, \\
V_{21, x}+2 i \lambda V_{21}+u_{2}\left(V_{22}-V_{11}\right)+V_{23}-V_{31}=0, \\
V_{23, x}+3 i \lambda V_{23}+V_{22}-V_{33}-u_{2} V_{13}+V_{21}=0, \\
V_{31, x}-i \lambda V_{31}+V_{33}-V_{11}-V_{21}+u_{2} V_{32}=0 \\
V_{32, x}-3 i \lambda V_{32}+V_{33}-V_{22}-V_{12}+u_{1} V_{31}=0,
\end{gathered}
$$

where each entry $V_{i j}$ is a Laurent expansion in $\lambda$ :

$$
V_{i j}=\sum_{k \geq 0} V_{i j, k-1} \lambda^{-k}, \quad 1 \leq i \leq 3,1 \leq j \leq 3,
$$

and $V_{11}, V_{22}, V_{33}$ satisfy $V_{11}+V_{22}+V_{33}=0$. A direct calculation shows that (8)-(16) implies the Lenard equation

$$
K G=\lambda J G, \quad G=\left(V_{12}, V_{13}, V_{21}, V_{23}, V_{31}, V_{32}, V_{22}, V_{33}\right)^{T} .
$$

Substituting Equation (17) into Equation (18) and collecting the same powers of $\lambda$, we get the following recursion relations:

$$
K G_{k-1}=J G_{k}, J G_{-1}=0
$$

where $G_{k}=\left(V_{12, k}, V_{13, k}, V_{21, k}, V_{23, k}, V_{31, k}, V_{32, k}, V_{22, k}, V_{33, k}\right)^{T}$. Since equation $J G^{-1}=0$ has the general solution

$$
G_{-1}=\alpha_{0} g_{-1}+\beta_{0} \widehat{g}_{-1}
$$

then $G_{j}$ can be expressed as

$$
\begin{aligned}
G_{k}= & \alpha_{0} g_{k}+\beta_{0} \widehat{g}_{k}+\cdots+\alpha_{k} g_{0}+\beta_{k} \widehat{g}_{0}+\alpha_{k+1} g_{-1} \\
& +\beta_{k+1} \widehat{g}_{-1}, \quad k \geq 0,
\end{aligned}
$$

where $\alpha_{k}$ and $\beta_{k}$ are arbitrary constants.

Consider the auxiliary problem:

$$
\psi_{t_{r}}=\tilde{V}^{(r)} \psi, \quad \tilde{V}^{(r)}=\left(\tilde{V}_{i j}^{(r)}\right)_{3 \times 3},
$$

where each entry $\tilde{V}_{i j}^{(r)}$ has the form $\tilde{V}_{i j}^{(r)}=\sum_{l=0}^{r} \tilde{V}_{i j, l-1} \lambda^{r-l}, 1$ $\leq i \leq 3,1 \leq j \leq 3$, and satisfies $\tilde{V}_{11}^{(r)}+\tilde{V}_{22}^{(r)}+\tilde{V}_{33}^{(r)}=0$. Then, we introduce $\tilde{G}_{s}=\left(\tilde{V}_{12, s}, \tilde{V}_{13, s}, \tilde{V}_{21, s}, \tilde{V}_{23, s}, \tilde{V}_{31, s}, \tilde{V}_{32, s}, \tilde{V}_{22, s}\right.$, $\left.\tilde{V}_{33, s}\right)^{T}$, which is determined by $\tilde{G}_{s}=\tilde{\alpha}_{0} g_{s}+\tilde{\beta}_{0} \widehat{g}_{s}+\cdots+\tilde{\alpha}_{s}$ $g_{0}+\tilde{\beta}_{s} \widehat{g}_{0}+\tilde{\alpha}_{s+1} g_{-1}+\tilde{\beta}_{s+1} \widehat{g}_{-1},-1 \leq s \leq r-1, \tilde{\alpha}_{s}$ and $\tilde{\beta}_{s}$ are arbitrary constants. It is easy to find that $\tilde{G}_{s}$ satisfies the Lenard equation $K \tilde{G}_{s}=J \tilde{G}_{s+1}, J \tilde{G}_{-1}=0$.

Then, from the compatibility condition $U_{t_{r}}-\tilde{V}_{x}^{(r)}+[U$, $\left.\tilde{V}^{(r)}\right]=0$ of Equations (2) and (22), we have

$$
u_{t_{r}}=X_{r}, \quad r \geq 0
$$

where the vector fields $X_{l}=X_{l}\left(u ; \underline{\alpha}^{(l)}, \underline{\beta}^{(l)}\right)=P\left(K \tilde{G}_{l-1}\right)=$ $P\left(J \tilde{G}_{l}\right)$, the constant vectors $\underline{\alpha}^{(l)}=\left(\tilde{\alpha}_{0}, \cdots, \tilde{\alpha}_{l}\right), \underline{\beta}^{(l)}=\left(\tilde{\beta}_{0}, \cdots\right.$, $\left.\tilde{\beta}_{l}\right)$, and the projective map $\gamma=\left(\gamma^{1}, \gamma^{2}, \gamma^{3}, \gamma^{4}, \gamma^{5}, \gamma^{6}, \gamma^{7}, \gamma^{8}\right)^{T}$ $\rightarrow\left(\gamma^{1}, \gamma^{2}\right)$. The third member in the hierarchy (22) is (for convenience, we take $\tilde{\alpha}_{1}=\tilde{\beta}_{1}=0$ )

$$
\begin{aligned}
u_{1, t_{2}}= & \tilde{\alpha}_{0}\left(\frac{1}{2} u_{1, x x}-\frac{26}{9} u_{1}-u_{1}^{2} u_{2}\right) \\
& +\tilde{\beta}_{0}\left(\frac{1}{4} u_{1, x x}-\frac{1}{9} u_{1}-\frac{1}{2} u_{1}^{2} u_{2}\right), \\
u_{2, t_{2}}= & \tilde{\alpha}_{0}\left(-\frac{1}{2} u_{2, x x}+\frac{26}{9} u_{2}+u_{2}^{2} u_{1}\right) \\
& +\tilde{\beta}_{0}\left(-\frac{1}{4} u_{2, x x}+\frac{1}{9} u_{2}+\frac{1}{2} u_{2}^{2} u_{1}\right) .
\end{aligned}
$$

Taking $\tilde{\alpha}_{0}=-11, \tilde{\alpha}_{0}=70$ in system (23), we have

$$
\begin{gathered}
u_{1, t_{2}}=12 u_{1, x x}+24\left(u_{1}-u_{1}^{2} u_{2}\right), \\
u_{2, t_{2}}=-12 u_{2, x x}-24\left(u_{2}-u_{2}^{2} u_{1}\right),
\end{gathered}
$$

which is called the CCIRD equation compared with Equation (0.4) in Ref. [4].

\section{The Stationary Baker-Akhiezer Function}

In the section, we are devoted to detailed study of the stationary Baker-Akhiezer function and the associated meromorphic functions. Then, the system of Dubrovin-type differential equations is derived. Let us consider the stationary CCIRD hierarchy: $X_{n}=0, n \geq 0$, which is equivalent to the stationary zero-curvature equation, $V_{x}^{(n)}=\left[U, V^{(n)}\right]$, $V^{(n)}=\left(\lambda^{n} V\right)_{+}=\left(V_{i, j}^{(n)}\right)_{3 \times 3}, V_{i, j}^{(n)}=\sum_{k=0}^{n} V_{i j, k-1} \lambda^{n-k}$, and $V_{i j, k-1}$ determined by (21). It is easy to verify that the matrix $y I-$ $V^{(n)}$ also satisfies the stationary zero-curvature equation. Then, the characteristic polynomial of Lax matrix $V^{(n)}, \mathscr{F}_{m}$ $(\lambda, y)=\operatorname{det}\left(y I-V^{(n)}\right)$, is independent of variable $x$ with the expansion

$$
\operatorname{det}\left(y I-V^{(n)}\right)=y^{3}+S_{m}(\lambda) y-T_{m}(\lambda),
$$


where $S_{m}(\lambda)$ and $T_{m}(\lambda)$ are polynomials with constant coefficients of $\lambda$ :

$$
\begin{aligned}
S_{m}(\lambda)= & \sum_{1 \leq i<j \leq 3}\left|\begin{array}{cc}
V_{i i}^{(n)} & V_{i j}^{(n)} \\
V_{j i}^{(n)} & V_{j j}^{(n)}
\end{array}\right|=-\left(\alpha_{0}^{2}+\beta_{0}^{2}+\alpha_{0} \beta_{0}\right) \lambda^{2 n} \\
& -\left(2 \alpha_{0} \alpha_{1}+2 \beta_{0} \beta_{1}+\alpha_{0} \beta_{1}+\alpha_{1} \beta_{0}\right) \lambda^{2 n-1}+\cdots, \\
T_{m}(\lambda)= & \left|\begin{array}{lll}
V_{11}^{(n)} & V_{12}^{(n)} & V_{13}^{(n)} \\
V_{21}^{(n)} & V_{22}^{(n)} & V_{23}^{(n)} \\
V_{31}^{(n)} & V_{32}^{(n)} & V_{33}^{(n)}
\end{array}\right|=-\alpha_{0} \beta_{0}\left(\alpha_{0}+\beta_{0}\right) \lambda^{3 n} \\
& -\left[\left(\alpha_{0}+\beta_{0}\right)\left(\alpha_{0} \beta_{1}+\alpha_{1} \beta_{0}\right)+\alpha_{0} \beta_{0}\left(\alpha_{1}+\beta_{1}\right)\right] \lambda^{3 n-1}+\cdots
\end{aligned}
$$

It is easy to find that $T_{m}(\lambda)$ is a polynomial of degree $3 n$ with respect to $\lambda$ as $\alpha_{0} \beta_{0}\left(\alpha_{0}+\beta_{0}\right) \neq 0$. Then, $\mathscr{F}_{m}(\lambda, y)=0$ naturally leads to a trigonal curve

$$
\mathscr{K}_{m-2}: \mathfrak{F}_{m}(\lambda, y)=y^{3}+S_{m}(\lambda) y-T_{m}(\lambda)=0,
$$

with $m=3 n$. For convenience, we denote the compactification of the curve $\mathscr{K}_{m-2}$ by the same symbol $\mathscr{K}_{m-2}$. Hence, $\mathscr{K}_{m-2}$ becomes a three-sheeted Riemann surface of arithmetic genus $m-2$ if it is nonsingular or smooth. Here, the meaning of nonsingular is that at each point $P^{\prime}=\left(\lambda^{\prime}, y^{\prime}\right) \in \mathscr{K}_{m-2},\left.\left(\partial \mathscr{F}_{m}(\lambda, y) / \partial \lambda, \partial \mathscr{F}_{m}(\lambda, y) / \partial y\right)\right|_{(\lambda, y)=(\lambda}{ }^{\prime}$ ,$\left.y^{\prime}\right) \neq 0$ holds. For $m \geq 4$, these curves are typically nonhyperelliptic. Point $P$ on $\mathscr{K}_{m-2}$ is represented as pairs $P=(\lambda, y(P))$ satisfying (29) along with $P_{\infty_{j}}=\left(\infty, \infty_{j}\right), j=1,2,3$, the three different points at infinity, which can be computed from the curve $y^{3}+S_{m}(\lambda) y-T_{m}(\lambda)=0$ by choosing $\lambda=\zeta^{-1}$. The complex structure on $\mathscr{K}_{m-2}$ is defined in the usual way by introducing local coordinate $\zeta_{Q}=\lambda-\lambda_{Q}$ near point $Q=$ $\left(\lambda_{Q}, y(Q)\right) \in \mathscr{K}_{m-2}$ which is neither branch nor singular point of $\mathscr{K}_{m-2}$ except the three points $P_{\mathrm{O}_{1}}, P_{\mathrm{\infty}_{2}}, P_{\mathrm{O}_{3}}$, at infinity with local coordinate $\lambda=\zeta^{-1}$ and local coordinate $\zeta_{Q_{0}}=\left(\lambda-\lambda_{Q_{0}}\right)^{-1 / 3}$ near branch or singular point $Q_{0}=\left(\lambda_{Q_{0}}\right.$, $\left.y\left(Q_{0}\right)\right) \in \mathscr{K}_{m-2}$.

Next, we shall define the meromorphic functions $\phi_{2}(P, x)$ and $\phi_{3}(P, x)$ on $\mathscr{K}_{m-2}$ as follows:

$$
\begin{array}{ll}
\phi_{2}(P, x)=\frac{\psi_{2}\left(P, x, x_{0}\right)}{\psi_{1}\left(P, x, x_{0}\right)}, & P \in \mathscr{K}_{m-2}, x \in \mathbb{C}, \\
\phi_{3}(P, x)=\frac{\psi_{3}\left(P, x, x_{0}\right)}{\psi_{1}\left(P, x, x_{0}\right)}, & P \in \mathscr{K}_{m-2}, x \in \mathbb{C},
\end{array}
$$

with the stationary Baker-Akhiezer function $\psi\left(P, x, x_{0}\right)$ defined by

$$
\begin{array}{r}
\psi_{x}\left(P, x, x_{0}\right)=U(u(x) ; \lambda(P)) \psi\left(P, x, x_{0}\right), \\
V^{(n)}(u(x) ; \lambda(P)) \psi\left(P, x, x_{0}\right)=y(P) \psi\left(P, x, x_{0}\right),
\end{array}
$$

$\psi_{1}\left(P, x_{0}, x_{0}\right)=1, \quad P=(\lambda, y) \in \mathscr{K}_{m-2} \backslash\left\{P_{\infty_{1}}, P_{\infty_{2}}, P_{\infty_{3}}\right\}, x \in \mathbb{C}$.

By using (31)-(33), a direct calculation gives that

$$
\begin{gathered}
\phi_{2}=\frac{F_{m-2}}{y^{2} V_{23}^{(n)}-y C_{m}+D_{m}}=\frac{y^{2} V_{13}^{(n)}-y A_{m}+B_{m}}{E_{m-2}}=\frac{y V_{23}^{(n)}+C_{m}}{y V_{13}^{(n)}+A_{m}}, \\
\phi_{3}=\frac{\mathscr{F}_{m-2}}{y^{2} V_{32}^{(n)}-y \mathscr{C}_{m}+\mathscr{D}_{m}}=\frac{y^{2} V_{12}^{(n)}-y \mathscr{A}_{m}+\mathscr{B}_{m}}{-E_{m-2}}=\frac{y V_{32}^{(n)}+\mathscr{C}_{m}}{y V_{12}^{(n)}+\mathscr{A}_{m}},
\end{gathered}
$$

where

$$
\begin{gathered}
A_{m}=V_{12}^{(n)} V_{23}^{(n)}-V_{13}^{(n)} V_{22}^{(n)}, \\
B_{m}=V_{13}^{(n)}\left(V_{11}^{(n)} V_{33}^{(n)}-V_{13}^{(n)} V_{31}^{(n)}\right)+V_{12}^{(n)}\left(V_{11}^{(n)} V_{23}^{(\mathrm{n})}-V_{13}^{(n)} V_{21}^{(n)}\right), \\
C_{m}=V_{13}^{(n)} V_{21}^{(n)}-V_{11}^{(n)} V_{23}^{(n)}, \\
D_{m}=V_{23}^{(n)}\left(V_{22}^{(n)} V_{33}^{(n)}-V_{23}^{(n)} V_{32}^{(n)}\right) \\
+V_{21}^{(n)}\left(V_{13}^{(n)} V_{22}^{(n)}-V_{12}^{(n)} V_{23}^{(n)}\right), \\
\mathscr{A}_{m}=V_{13}^{(n)} V_{32}^{(n)}-V_{12}^{(n)} V_{33}^{(n)}, \\
\mathscr{B}_{m}=V_{13}^{(n)}\left(V_{11}^{(n)} V_{22}^{(n)}-V_{12}^{(n)} V_{21}^{(n)}\right) \\
+V_{13}^{(n)}\left(V_{11}^{(n)} V_{32}^{(n)}-V_{12}^{(n)} V_{31}^{(n)}\right), \\
\mathscr{F}_{m-2}=\left(V_{12}^{(n)}\right)^{2} V_{32}^{(n)}+V_{31}^{(n)} V_{32}^{(n)}\left(V_{11}^{(n)}-V_{22}^{(n)}\right)-\left(V_{11}^{(n)} V_{32}^{(n)},\right. \\
\left.E_{m-2}^{(n)}\right)^{2} V_{12}^{(n)} . \\
\mathscr{D}_{m}=V_{32}^{(n)}\left(V_{22}^{(n)} V_{33}^{(n)}-V_{23}^{(n)} V_{32}^{(n)}\right) \\
+V_{31}^{(n)}\left(V_{12}^{(n)} V_{33}^{(n)}-V_{13}^{(n)} V_{32}^{(n)}\right), \\
\left.V_{13}^{(n)}\right)^{2} V_{32}^{(n)}+V_{12}^{(n)} V_{13}^{(n)}\left(V_{22}^{(n)}-V_{33}^{(n)}\right)-\left(V_{12}^{(n)}\right)^{2} V_{23}^{(n)}, \\
(37) \\
(n) \\
(n)
\end{gathered}
$$

Through straightforward calculations, we obtain some main interrelationships among polynomials $A_{m}, \cdots, D_{m}, \mathscr{A}_{m}$, $\cdots, \mathscr{D}_{m}, E_{m-2}, F_{m-2}, \mathscr{F}_{m-2}, S_{m}, T_{m}$, some of which are summarized below: 


$$
\begin{aligned}
& V_{13}^{(n)} F_{m-2}=V_{23}^{(n)} D_{m}-S_{m}\left(V_{23}^{(n)}\right)^{2}-C_{m}^{2}, \\
& A_{m} F_{m-2}=T_{m}\left(V_{23}^{(n)}\right)^{2}+C_{m} D_{m} \\
& V_{23}^{(n)} E_{m-2}=V_{13}^{(n)} B_{m}-S_{m}\left(V_{13}^{(n)}\right)^{2}-A_{m}^{2}, \\
& C_{m} E_{m-2}=T_{m}\left(V_{13}^{(n)}\right)^{2}+A_{m} B_{m}, \\
& V_{23}^{(n)} B_{m}+V_{13}^{(n)} D_{m}-V_{13}^{(n)} V_{23}^{(n)} S_{m}+A_{m} C_{m}=0, \\
& V_{13}^{(n)} V_{23}^{(n)} T_{m}+V_{23}^{(n)} A_{m} S_{m}+V_{13}^{(n)} C_{m} S_{m}-B_{m} C_{m}-A_{m} D_{m}=0 \text {, } \\
& V_{23}^{(n)} A_{m} T_{m}+V_{13}^{(n)} C_{m} T_{m}+E_{m-2} F_{m-2}-B_{m} D_{m}=0, \\
& V_{12}^{(n)} \mathscr{F}_{m-2}=V_{32}^{(n)} \mathscr{D}_{m}-S_{m}\left(V_{32}^{(n)}\right)^{2}-\mathscr{C}_{m}^{2}, \\
& \mathscr{A}_{m} \mathscr{F}_{m-2}=T_{m}\left(V_{32}^{(n)}\right)^{2}+\mathscr{C}_{m} \mathscr{D}_{m} \\
& -V_{32}^{(n)} E_{m-2}=V_{12}^{(n)} \mathscr{B}_{m}-S_{m}\left(V_{12}^{(n)}\right)^{2}-\mathscr{A}_{m}^{2}, \\
& -\mathscr{C}_{m} E_{m-2}=T_{m}\left(V_{12}^{(n)}\right)^{2}+\mathscr{A}_{m} \mathscr{B}_{m}, \\
& V_{32}^{(n)} \mathscr{B}_{m}+V_{12}^{(n)} \mathscr{D}_{m}-V_{12}^{(n)} V_{32}^{(n)} S_{m}+\mathscr{A}_{m} \mathscr{C}_{m}=0, \\
& V_{12}^{(n)} V_{32}^{(n)} T_{m}+V_{32}^{(n)} \mathscr{A}_{m} S_{m}+V_{13}^{(n)} \mathscr{C}_{m} S_{m}-\mathscr{B}_{m} \mathscr{C}_{m}-\mathscr{A}_{m} \mathscr{D}_{m}=0 \text {, } \\
& V_{32}^{(n)} \mathscr{A}_{m} T_{m}+V_{12}^{(n)} \mathscr{C}_{m} T_{m}+E_{m-2} \mathscr{F}_{m-2}-\mathscr{B}_{m} \mathscr{D}_{m}=0, \\
& E_{m-2, x}=i \lambda E_{m-2}-u_{1}\left(2 V_{13}^{(n)} S_{m}-3 B_{m}\right)+2 V_{12}^{(n)} S_{m}-3 \mathscr{B}_{m} \text {, } \\
& V_{23}^{(n)} F_{m-2, x}=\left(V_{21}-u_{2} V_{23}^{(n)}\right)\left(2 V_{23}^{(n)} S_{m}-3 D_{m}\right) \\
& -\left(5 i \lambda V_{23}^{(n)}+3 V_{22}^{(n)}\right) F_{m-2} \text {, } \\
& V_{32}^{(n)} \mathscr{F}_{m-2, x}=\left(V_{31}-V_{32}^{(n)}\right)\left(2 V_{23}^{(n)} S_{m}-3 \mathscr{D}_{m}\right) \\
& +\left(4 i \lambda V_{32}^{(n)}-3 V_{33}^{(n)}\right) \mathscr{F}_{m-2} .
\end{aligned}
$$

By observing Equations (21) and (38), one infers that $E_{m-2}$, $F_{m-2}$, and $\mathscr{F}_{m-2}$ are polynomials with respect to $\lambda$ of degree $m-2$. Therefore, we may write them in the following forms:

$$
E_{m-2}(\lambda, x)=\frac{\left(\alpha_{0}-\beta_{0}\right)\left(\alpha_{0}+2 \beta_{0}\right)\left(2 \alpha_{0}+\beta_{0}\right)}{2} u_{1} \prod_{j=1}^{m-2}\left(\lambda-\mu_{j}(x)\right),
$$

$$
F_{m-2}(\lambda, x)=\frac{\left(\alpha_{0}-\beta_{0}\right)\left(\alpha_{0}+2 \beta_{0}\right)\left(2 \alpha_{0}+\beta_{0}\right)}{6} u_{2} \prod_{j=1}^{m-2}\left(\lambda-v_{j}(x)\right),
$$

$$
\mathscr{F}_{m-2}(\lambda, x)=\frac{\left(\beta_{0}-\alpha_{0}\right)\left(\alpha_{0}+2 \beta_{0}\right)\left(2 \alpha_{0}+\beta_{0}\right)}{3} \prod_{j=1}^{m-2}\left(\lambda-\xi_{j}(x)\right),
$$

where $\left(\alpha_{0}-\beta_{0}\right)\left(\alpha_{0}+2 \beta_{0}\right)\left(2 \alpha_{0}+\beta_{0}\right) \neq 0$.

Define

$$
\begin{gathered}
\widehat{\mu}_{i}(x)=\left(\mu_{j}(x), y\left(\widehat{\mu}_{j}(x)\right)\right)=\left(\mu_{j}(x),-\frac{A_{m}\left(\mu_{j}(x), x\right)}{V_{13}^{(n)}\left(\mu_{j}(x), x\right)}\right) \\
=\left(\mu_{j}(x),-\frac{\mathscr{A}_{m}\left(\mu_{j}(x), x\right)}{V_{12}^{(n)}\left(\mu_{j}(x), x\right)}\right) \in \mathscr{K}_{m-2}, \\
\widehat{v}_{j}(x)=\left(v_{j}(x), y\left(\widehat{v}_{j}(x)\right)\right)=\left(v_{j}(x),-\frac{C_{m}\left(v_{j}(x), x\right)}{V_{23}^{(n)}\left(v_{j}(x), x\right)}\right) \in \mathscr{K}_{m-2}, \\
\widehat{\xi}_{j}(x)=\left(\xi_{j}(x), y\left(\widehat{\xi}_{j}(x)\right)\right)=\left(\xi_{j}(x),-\frac{\mathscr{C}_{m}\left(\xi_{j}(x), x\right)}{V_{32}^{(n)}\left(\xi_{j}(x), x\right)}\right) \in \mathscr{K}_{m-2}, \\
1 \leq j \leq m-2, x \in \mathbb{C} .
\end{gathered}
$$

In order to more distinctly put forward the properties of $\phi_{2}(P, x), \phi_{3}(P, x)$, and $\psi_{1}\left(P, x, x_{0}\right)$, we introduce the holomorphic map $*$, changing sheets, which is defined by

$$
*:\left\{\begin{aligned}
\mathscr{K}_{m-1} \rightarrow \mathscr{K}_{m-1}, \\
P=\left(\lambda, y_{i}(\lambda)\right) \rightarrow P^{*}=\left(\lambda, y_{i+1(\bmod 3)}(\lambda)\right), \quad i=0,1,2, \\
P^{* *}:=\left(P^{*}\right)^{*}, \text { etc. }
\end{aligned}\right.
$$

where $y_{j}(\lambda), j=0,1,2$, satisfy $\mathscr{F}_{m}(\lambda, y)=0$, that is,

$$
\left(y-y_{0}(\lambda)\right)\left(y-y_{1}(\lambda)\right)\left(y-y_{2}(\lambda)\right)=y^{3}+S_{m}(\lambda) y-T_{m}(\lambda)=0 .
$$

Furthermore, the positive divisors on $\mathscr{K}_{m-2}$ of degree $m-2$ are defined as

$$
\mathscr{D}_{P_{1}, \cdots, P_{m-2}}=\left\{\begin{array}{l}
\mathscr{K}_{m-2} \rightarrow \mathbb{N}_{0}, \\
P \rightarrow \mathscr{D}_{P_{1}, \cdots, P_{m-2}}= \begin{cases}0, & \text { if } P \notin\left\{P_{1}, \cdots, P_{m-2}\right\}, \\
k, & \text { if } P \text { occurs } k \text { times } \in\left\{P_{1}, \cdots, P_{m-2}\right\},\end{cases}
\end{array}\right.
$$

with $\mathbb{N}_{0}=\mathbb{N} \cup\{0\}$.

Further properties of $\phi_{2}(P, x), \phi_{3}(P, x)$, and $\psi_{1}\left(P, x, x_{0}\right)$ are summarized as follows:

$$
\begin{aligned}
& \phi_{2, x}(P, x)+u_{1} \phi_{2}^{2}(P, x)+\phi_{2}(P, x) \phi_{3}(P, x)+2 i \lambda \phi_{2}(P, x) \\
& \quad-\phi_{3}(P, x)-u_{2}=0,
\end{aligned}
$$




$$
\begin{aligned}
& \phi_{3, x}(P, x)+\phi_{3}^{2}(P, x)+u_{1} \phi_{2}(P, x) \phi_{3}(P, x)-i \lambda \phi_{3}(P, x) \\
& -\phi_{2}(P, x)-1=0 \text {, } \\
& \phi_{2}(P, x) \phi_{2}\left(P^{*}, x\right) \phi_{2}\left(P^{* *}, x\right)=-\frac{F_{m-2}(\lambda, x)}{E_{m-2}(\lambda, x)}, \\
& \phi_{3}(P, x) \phi_{3}\left(P^{*}, x\right) \phi_{3}\left(P^{* *}, x\right)=\frac{\mathscr{F}_{m-2}(\lambda, x)}{E_{m-2}(\lambda, x)}, \\
& \phi_{2}(P, x)+\phi_{2}\left(P^{*}, x\right)+\phi_{2}\left(P^{* *}, x\right) \\
& =-\frac{2 V_{13}^{(n)}(\lambda, x) S_{m}(\lambda)-3 B_{m}(\lambda, x)}{E_{m-2}(\lambda, x)} \\
& =\left[\frac{E_{m-2, x}(\lambda, x)}{E_{m-2}(\lambda, x)}-i \lambda+3 \frac{V_{11}^{(n)}(\lambda, x)}{V_{13}^{(n)}(\lambda, x)}\right] \\
& \times \frac{V_{13}^{(n)}(\lambda, x)}{u_{1}(x) V_{13}^{(n)}(\lambda, x)-V_{12}^{(n)}(\lambda, x)}, \\
& \phi_{3}(P, x)+\phi_{3}\left(P^{*}, x\right)+\phi_{3}\left(P^{* *}, x\right) \\
& =\frac{2 V_{12}^{(n)}(\lambda, x) S_{m}(\lambda)-3 \mathscr{B}_{m}(\lambda, x)}{E_{m-2}(\lambda, x)} \\
& =-\left[\frac{E_{m-2, x}(\lambda, x)}{E_{m-2}(\lambda, x)}-i \lambda+3 u_{1}(x) \frac{V_{11}^{(n)}(\lambda, x)}{V_{12}^{(n)}(\lambda, x)}\right] \\
& \times \frac{V_{12}^{(n)}(\lambda, x)}{u_{1}(x) V_{13}^{(\mathrm{n})}(\lambda, x)-V_{12}^{(n)}(\lambda, x)},
\end{aligned}
$$

$$
\begin{aligned}
& u_{1}\left[\phi_{2}(P, x)+\phi_{2}\left(P^{*}, x\right)+\phi_{2}\left(P^{* *}, x\right)\right] \\
& \quad+\phi_{3}(P, x)+\phi_{3}\left(P^{*}, x\right)+\phi_{3}\left(P^{* *}, x\right)=\frac{E_{m-2, x}(\lambda, x)}{E_{m-2}(\lambda, x)}-i \lambda,
\end{aligned}
$$

$$
\begin{aligned}
& \frac{1}{\phi_{2}(P, x)}+\frac{1}{\phi_{2}\left(P^{*}, x\right)}+\frac{1}{\phi_{2}\left(P^{* *}, x\right)} \\
& =\frac{\left[-5 i \lambda V_{23}^{(n)}(\lambda, x)-3 V_{22}^{(n)}(\lambda, x)\right] F_{m-2}(\lambda, x)-V_{23}^{(n)}(\lambda, x) F_{m-2, x}(\lambda, x)}{\left[V_{21}^{(n)}(\lambda, x)-u_{2} V_{23}^{(n)}(\lambda, x)\right] F_{m-2}(\lambda, x)},
\end{aligned}
$$

$$
\begin{aligned}
& \frac{1}{\phi_{3}(P, x)}+\frac{1}{\phi_{3}\left(P^{*}, x\right)}+\frac{1}{\phi_{3}\left(P^{* *}, x\right)} \\
& =\frac{\left[4 i \lambda V_{32}^{(n)}(\lambda, x)-3 V_{33}^{(n)}(\lambda, x)\right] \mathscr{F}_{m-2}(\lambda, x)-V_{32}^{(n)}(\lambda, x) \mathscr{F}_{m-2, x}(\lambda, x)}{\left[V_{31}^{(n)}(\lambda, x)-V_{32}^{(n)}(\lambda, x)\right] \mathscr{F}_{m-2}(\lambda, x)},
\end{aligned}
$$

$$
\begin{aligned}
& \frac{\phi_{2}(P, x)}{\phi_{3}(P, x)}+\frac{\phi_{2}\left(P^{*}, x\right)}{\phi_{3}\left(P^{*}, x\right)}+\frac{\phi_{2}\left(P^{* *}, x\right)}{\phi_{3}\left(P^{* *}, x\right)} \\
& =\frac{1}{V_{32}^{(n)}(\lambda, x)\left[V_{31}^{(n)}(\lambda, x)-V_{32}^{(n)}(\lambda, x)\right] \mathscr{F}_{m-2}(\lambda, x)} \\
& \quad \times\left\{V_{31}^{(n)}(\lambda, x) V_{32}^{(n)}(\lambda, x) \mathscr{F}_{m-2, x}(\lambda, x)+V_{31}^{(n)}(\lambda, x)\right. \\
& \left.\cdot\left[3 V_{33}^{(n)}(\lambda, x)-4 i \lambda V_{32}^{(n)}(\lambda, x)\right] \mathscr{F}_{m-2}(\lambda, x)\right\}-3 \frac{V_{33}^{(n)}(\lambda, x)}{V_{32}^{(n)}(\lambda, x)},
\end{aligned}
$$

$$
\begin{aligned}
& \frac{\phi_{3}(P, x)}{\phi_{2}(P, x)}+\frac{\phi_{3}\left(P^{*}, x\right)}{\phi_{2}\left(P^{*}, x\right)}+\frac{\phi_{3}\left(P^{* *}, x\right)}{\phi_{2}\left(P^{* *}, x\right)} \\
& =\frac{1}{V_{23}^{(n)}(\lambda, x)\left[V_{21}^{(n)}(\lambda, x)-u_{2} V_{23}^{(n)}(\lambda, x)\right] F_{m-2}(\lambda, x)} \\
& \quad \times\left\{V_{21}^{(n)}(\lambda, x) V_{23}^{(n)}(\lambda, x) F_{m-2, x}(\lambda, x)+V_{21}^{(n)}(\lambda, x)\right. \\
& \left.\quad \cdot\left[3 V_{22}^{(n)}(\lambda, x)-4 i \lambda V_{23}^{(n)}(\lambda, x)\right] F_{m-2}(\lambda, x)\right\}-3 \frac{V_{22}^{(n)}(\lambda, x)}{V_{23}^{(n)}(\lambda, x)},
\end{aligned}
$$

$$
\begin{gathered}
\psi_{1}\left(P, x, x_{0}\right)=\exp \left(\int_{x_{0}}^{x}\left[i \lambda+u_{1}\left(x^{\prime}\right) \phi_{2}\left(P, x^{\prime}\right)+\phi_{3}\left(P, x^{\prime}\right)\right] d x^{\prime}\right) \\
\psi_{1}\left(P, x, x_{0}\right) \psi_{1}\left(P^{*}, x, x_{0}\right) \psi_{1}\left(P^{* *}, x, x_{0}\right) \\
=\frac{E_{m-2}(\lambda, x)}{E_{m-2}\left(\lambda, x_{0}\right)} \exp \left(2 i \lambda\left(x-x_{0}\right)\right) .
\end{gathered}
$$

The dynamics of the zeros $\left\{\mu_{j}(x)\right\}_{j=1}^{m-2},\left\{v_{j}(x)\right\}_{j=1}^{m-2}$, and $\left\{\xi_{j}(x)\right\}_{j=1}^{m-2}$ of $E_{m-2}(\lambda, x), F_{m-2}(\lambda, x)$, and $\mathscr{F}_{m}(\lambda, x)$ are described according to Dubrovin-type equations as follows.

Lemma 1. Assume the zeros $\left\{\mu_{j}(x)\right\}_{j=1}^{m-2},\left\{\nu_{j}(x)\right\}_{j=1}^{m-2}$, and $\left\{\xi_{j}(x)\right\}_{j=1}^{m-2}$ of $E_{m-2}(\lambda, x), F_{m-2}(\lambda, x)$, and $\mathscr{F}_{m}(\lambda, x)$ remain distinct, respectively, and let $x \in \mathbb{C}$. Then, $\left\{\mu_{j}(x)\right\}_{j=1}^{m-2}$, $\left\{v_{j}(x)\right\}_{j=1}^{m-2}$, and $\left\{\xi_{j}(x)\right\}_{j=1}^{m-2}$ satisfy the system of differential equations

$$
\mu_{j, x}(x)=\frac{2\left[V_{12}^{(n)}\left(\mu_{j}(x), x\right)-u_{1} V_{13}^{(n)}\left(\mu_{j}(x), x\right)\right]\left[3 y^{2}\left(\widehat{\mu}_{j}(x)\right)+S_{m}\left(\mu_{j}(x)\right)\right]}{\left(\alpha_{0}-\beta_{0}\right)\left(\alpha_{0}+2 \beta_{0}\right)\left(2 \alpha_{0}+\beta_{0}\right) u_{1} \prod_{k=1}^{m-2} k \neq j},
$$

$$
v_{j, x}(x)=\frac{6\left[V_{21}^{(n)}\left(v_{j}(x), x\right)-u_{2} V_{23}^{(n)}\left(v_{j}(x), x\right)\right]\left[3 y^{2}\left(\widehat{v}_{j}(x)\right)+S_{m}\left(v_{j}(x)\right)\right]}{\left(\alpha_{0}-\beta_{0}\right)\left(\alpha_{0}+2 \beta_{0}\right)\left(2 \alpha_{0}+\beta_{0}\right) u_{2} \prod_{k=1}^{m-2} k \neq j\left(v_{j}(x)-v_{k}(x)\right)},
$$


$\xi_{j, x}(x)=-\frac{3\left[V_{31}^{(n)}\left(\xi_{j}(x), x\right)-V_{32}^{(n)}\left(\xi_{j}(x), x\right)\right]\left[3 y^{2}\left(\widehat{\xi}_{j}(x)\right)+S_{m}\left(\xi_{j}(x)\right)\right]}{\left(\alpha_{0}-\beta_{0}\right)\left(\alpha_{0}+2 \beta_{0}\right)\left(2 \alpha_{0}+\beta_{0}\right) \prod_{k=1}^{m-2} k \neq j\left(\xi_{j}(x)-\xi_{k}(x)\right)}, \quad 1 \leq j \leq m-2$

Proof. Substituting $\lambda=\mu_{j}(x)$ into (40) and (43), we have

$$
\begin{aligned}
& \left(V_{13}^{(n)}\left(\mu_{j}(x), x\right)\right)^{2} S_{m}\left(\mu_{j}(x)\right)-V_{13}^{(n)}\left(\mu_{j}(x), x\right) B_{m}\left(\mu_{j}(x)\right) \\
& \quad+A_{m}^{2}\left(\mu_{j}(x)\right)=0 \\
& \left(V_{12}^{(n)}\left(\mu_{j}(x), x\right)\right)^{2} S_{m}\left(\mu_{j}(x)\right)-V_{12}^{(n)}\left(\mu_{j}(x), x\right) \mathscr{B}_{m}\left(\mu_{j}(x)\right) \\
& +\mathscr{A}_{m}^{2}\left(\mu_{j}(x)\right)=0 .
\end{aligned}
$$

Then, we get

$$
\begin{aligned}
B_{m}\left(\mu_{j}(x), x\right) & =S_{m}\left(\mu_{j}(x)\right) V_{13}^{(n)}\left(\mu_{j}(x), x\right)+\frac{A_{m}^{2}\left(\mu_{j}(x), x\right)}{V_{13}^{(n)}\left(\mu_{j}(x), x\right)} \\
& =\left[S_{m}\left(\mu_{j}(x)\right)+y^{2}\left(\mu_{j}(x)\right)\right] V_{13}^{(n)}\left(\mu_{j}(x), x\right), \\
& (70) \\
\mathscr{B}_{m}\left(\mu_{j}(x), x\right) & =S_{m}\left(\mu_{j}(x)\right) V_{12}^{(n)}\left(\mu_{j}(x), x\right)+\frac{\mathscr{A}_{m}^{2}\left(\mu_{j}(x), x\right)}{V_{12}^{(n)}\left(\mu_{j}(x), x\right)} \\
& =\left[S_{m}\left(\mu_{j}(x)\right)+y^{2}\left(\mu_{j}(x)\right)\right] V_{12}^{(n)}\left(\mu_{j}(x), x\right) .
\end{aligned}
$$

Then, inserting $\lambda=\mu_{j}(x)$ into the first equation of (45) and in view of (70) and (71), we arrive at

$$
\begin{aligned}
E_{m-2, x}\left(\mu_{j}(x), x\right)= & {\left[u_{1} V_{13}^{(n)}\left(\mu_{j}(x), x\right)-V_{12}^{(n)}\left(\mu_{j}(x), x\right)\right] } \\
& \cdot\left[3 y^{2}\left(\widehat{\mu}_{j}(x)\right)+S_{m}\left(\mu_{j}(x)\right)\right] .
\end{aligned}
$$

On the other hand, differentiating (46) with respect to $x$, we find

$$
\begin{aligned}
E_{m-2, x}\left(\mu_{j}(x), x\right)= & \frac{\left(\beta_{0}-\alpha_{0}\right)\left(\alpha_{0}+2 \beta_{0}\right)\left(2 \alpha_{0}+\beta_{0}\right) u_{1}}{2} \\
& \cdot \mu_{j, x}(x) \prod_{k=1}^{m-2}\left(\mu_{j}(x)-\mu_{k}(x)\right) .
\end{aligned}
$$

Comparing (72) and (73), we derive (66). Similarly, we can prove that Equations (67) and (68) hold.

\section{Algebro-Geometric Solutions of the Stationary CCIRD Hierarchy}

In the section, we continue our study of the stationary CCIRD hierarchy and will obtain explicit Riemann theta function representations for the two meromorphic functions $\phi_{2}(P, x), \phi_{3}(P, x)$, the Baker-Akhiezer function $\psi_{1}\left(P, x, x_{0}\right)$, and the algebro-geometric solutions $u_{1}$ and $u_{2}$ for the CCIRD hierarchy. By introducing the local coordinate $\zeta=\lambda^{-1}$ near $P_{\infty_{1}}, P_{\infty_{2}}, P_{\infty_{3}} \in \mathscr{K}_{m-2}$, we have the following lemma.

Lemma 2. Suppose that $u$ satisfies the nth stationary CCIRD system $X_{n}=0$. Moreover, let $P \in \mathscr{K}_{m-2} \backslash\left\{P_{\infty_{1}}, P_{\infty_{2}}, P_{\infty_{3}}\right\}$, $\left(x, x_{0}\right) \in \mathbb{C}^{2}$. Then,

$\phi_{2}(P, x)= \begin{cases}-2 i u_{1}^{-1}(x) \zeta^{-1}+\frac{u_{1, x}(x)}{u_{1}^{2}(x)}-\frac{2}{3} u_{1}^{-2}(x)+O(\zeta), & \text { as } P \rightarrow P_{\mathrm{\infty}_{1}}, \\ \frac{1}{3}+O(\zeta), & \text { as } P \rightarrow P_{\mathrm{\infty}_{2}}, \\ -\frac{i}{2} u_{2}(x) \zeta+\left[\frac{1}{2}+\frac{1}{4} u_{2, x}(x)\right] \zeta^{2}+O\left(\zeta^{3}\right), & \text { as } P \rightarrow P_{\mathrm{\infty}_{3}},\end{cases}$

$$
\begin{gathered}
\phi_{3}(P, x)= \begin{cases}\frac{2}{3} u_{1}^{-1}(x)+O(\zeta), & \text { as } P \rightarrow P_{\infty_{1}}, \\
i \zeta^{-1}-\frac{1}{3} u_{1}(x)+O(\zeta), & \text { as } \rightarrow P_{\infty_{2}}, \\
i \zeta+\frac{1}{2} u_{2}(x) \zeta^{2}+O\left(\zeta^{3}\right), & \text { as } P \rightarrow P_{\infty_{3}},\end{cases} \\
\psi_{1}(P, x)= \begin{cases}\frac{u_{1}(x)}{u_{1}\left(x_{0}\right)} \exp \left(-i\left(x-x_{0}\right) \zeta^{-1}+O(\zeta)\right), & \text { as } P \rightarrow P_{\infty_{1}}, \\
\exp \left(2 i\left(x-x_{0}\right) \zeta^{-1}+O(\zeta)\right), & \text { as } P \rightarrow P_{\infty_{2}}, \\
\exp \left(i\left(x-x_{0}\right) \zeta^{-1}+O(\zeta)\right), & \text { as } P \rightarrow P_{\infty_{3}} .\end{cases}
\end{gathered}
$$

Proof. Substituting the three sets of ansatz

$$
\begin{array}{ccc}
\phi_{2}(P, x) \underset{\zeta \rightarrow 0}{=} \kappa_{1,-1} \zeta^{-1}+\kappa_{1,0}+O(\zeta), & \phi_{3}(P, x) \underset{\zeta \rightarrow 0}{=} \chi_{1,0}+O(\zeta), & \operatorname{near} P_{\infty_{1}}, \\
\phi_{2}(P, x) \underset{\zeta \rightarrow 0}{=} \kappa_{2,0}+O(\zeta), & \phi_{3}(P, x)=\underset{\zeta \rightarrow 0}{=} \kappa_{2,-1} \zeta^{-1}+\chi_{2,0}+O(\zeta), & \text { near } P_{\infty_{2}}, \\
\phi_{2}(P, x) \underset{\zeta \rightarrow 0}{=} \kappa_{3,1} \zeta+\kappa_{3,2} \zeta^{2}+O\left(\zeta^{3}\right), & \phi_{3}(P, x) \underset{\zeta \rightarrow 0}{=} \chi_{3,1} \zeta+\chi_{3,2} \zeta^{2}+O\left(\zeta^{3}\right), & \text { near } P_{\infty_{3}},
\end{array}
$$

into Riccati-type Equations (53) and (54), and comparing the coefficients of the same powers of $\zeta$, we derive (74) and (75). Equation (76) then follows from inserting (74) and (75) into (64).

One infers from Equations (34), (35), (74), and (75) that the divisors $\left(\phi_{2}(P, x)\right)$ and $\left(\phi_{3}(P, x)\right)$ of $\phi_{2}(P, x)$ and $\phi_{3}(P, x)$ are as follows: 


$$
\begin{aligned}
\left(\phi_{2}(P, x)\right)= & \mathfrak{D}_{P_{\mathrm{c}_{3}}, \widehat{v}_{1}(x), \cdots, \widehat{v}_{m-2}(x)}(P) \\
& -\mathfrak{D}_{P_{\infty_{1}}, \widehat{\mu}_{1}(x), \cdots, \widehat{\mu}_{m-2}(x)}(P), \\
\left(\phi_{3}(P, x)\right)= & \mathfrak{D}_{P_{\mathrm{\infty}_{3}}, \widehat{\xi}_{1}(x), \cdots, \widehat{\xi}_{m-2}(x)}(P) \\
& -\mathfrak{D}_{P_{\mathrm{\infty}_{2}}, \widehat{\mu}_{1}(x), \cdots, \widehat{\mu}_{m-2}(x)}(P),
\end{aligned}
$$

which means that $P_{\infty_{3}}, \widehat{v}_{1}(x), \cdots, \widehat{v}_{m-2}(x)$ are $m-1$ zeros of $\phi_{2}(P, x)$ and $P_{\infty_{1}}, \widehat{\mu}_{1}(x), \cdots, \widehat{\mu}_{m-2}(x)$ its $m-1$ poles, $P_{\infty_{3}}$, $\widehat{\xi}_{1}(x), \cdots, \widehat{\xi}_{m-2}(x)$ are $m-1$ zeros of $\phi_{3}(P, x)$ and $P_{\infty_{2}}, \widehat{\mu}_{1}($ $x), \cdots, \widehat{\mu}_{m-2}(x)$ its $m-1$ poles.

A tedious calculation reveals that the asymptotic behaviors of $y(P)$ and $S_{m}$ near $P_{\infty_{1}}, P_{\infty_{2}}, P_{\infty_{3}}$ are given as

$$
y(P) \underset{\zeta \rightarrow 0}{=} \begin{cases}\zeta^{-n}\left[\alpha_{0}+\alpha_{1} \zeta+O\left(\zeta^{2}\right)\right], & \text { as } P \rightarrow P_{\infty_{1}}, \\ \zeta^{-n}\left[\beta_{0}+\beta_{1} \zeta+O\left(\zeta^{2}\right)\right], & \text { as } P \rightarrow P_{\infty_{2}}, \\ -\zeta^{-n}\left[\alpha_{0}+\beta_{0}+\left(\alpha_{1}+\beta_{1}\right) \zeta+O\left(\zeta^{2}\right)\right], & \text { as } P \rightarrow P_{\infty_{3}}\end{cases}
$$$$
S_{m_{\zeta \rightarrow 0}=}^{=} \zeta^{-2 n}\left\{\left[\alpha_{0}^{2}+\beta_{0}^{2}+\alpha_{0} \beta_{0}+2\left[\left(\alpha_{0}+\beta_{0}\right)\left(\alpha_{1}+\beta_{1}\right)-\alpha_{0} \beta_{1}-\alpha_{1} \beta_{0}\right] \zeta+O\left(\zeta^{2}\right)\right]\right\},
$$

$$
\text { as } P \rightarrow P_{\infty_{j}}, j=1,2,3 \text {. }
$$

Equip the Riemann surface $\mathscr{K}_{m-2}$ with an appropriate fixed homology basis $\left\{a_{j}, b_{j}\right\}_{j=1}^{m-2}$, in such a way that the intersection matrix of cycles satisfies $a_{j} \circ b_{k}=\delta_{j, k}, a_{j} \circ a_{k}=0, b_{j} \circ$ $b_{k}=0, j, k=1, \cdots, m-2$. For the present, we introduce the holomorphic differentials $\omega_{l}(P)$ on $\mathscr{K}_{m-2}$ defined by

$$
\omega_{l}(P)=\frac{1}{3 y^{2}(P)+S_{m}} \begin{cases}\lambda^{l-1} d \lambda, & 1 \leq l \leq 2 n-1, \\ y(P) \lambda^{l+n-m} d \lambda, & 2 n \leq l \leq m-2 .\end{cases}
$$

By using the basis $a_{j}$ and $b_{j}$, the matrices $A$ and $B$ can be constructed from

$$
\begin{aligned}
& A_{j k}=\int_{a_{k}} \omega_{j}, \\
& B_{j k}=\int_{b_{k}} \omega_{j},
\end{aligned}
$$

and it is possible to show that matrices $A$ and $B$ are invertible. Now, we define the matrices $C$ and $\tau$ by $C=A^{-1}, \tau=A^{-1} B$. One can see that matrix $\tau=\left(\tau_{i j}\right)_{(m-1) \times(m-1)}$ is symmetric, and it has a positive definite imaginary part. If we normalize $\omega_{l}$ into the new basis $\omega_{j}$,

$$
\omega_{j}=\sum_{l=1}^{m-2} C_{j l} \omega_{l}
$$

then we have $\int_{a_{k}} \omega_{j}=\delta_{j k}, \int_{b_{k}} \omega_{j}=\tau_{j k}, j, k=1, \cdots, m-2$.

Then, the Laurent expansion of (83) near $\left\{P_{\infty_{1}}, P_{\infty_{2}}\right.$, $\left.P_{\infty_{3}}\right\}$ yields the following results:

$$
\omega=\left(\omega_{1}, \cdots, \omega_{j}, \cdots, \omega_{m-2}\right),
$$

$$
\omega_{j}= \begin{cases}\left(-\frac{C_{j, 2 n-1}+\alpha_{0} C_{j, m-2}}{\left(\alpha_{0}-\beta_{0}\right)\left(2 \alpha_{0}+\beta_{0}\right)}+O(\zeta)\right) d \zeta, & P \rightarrow P_{\infty_{1}}, \\ \left(\frac{C_{j, 2 n-1}+\beta_{0} C_{j, m-2}}{\left(\alpha_{0}-\beta_{0}\right)\left(\alpha_{0}+2 \beta_{0}\right)}+O(\zeta)\right) d \zeta, & P \rightarrow P_{\infty_{2}} \\ \left(-\frac{C_{j, 2 n-1}-\left(\alpha_{0}+\beta_{0}\right) C_{j, m-2}}{\left(\alpha_{0}+2 \beta_{0}\right)\left(2 \alpha_{0}+\beta_{0}\right)}+O(\zeta)\right) d \zeta, & P \rightarrow P_{\infty_{3}}\end{cases}
$$

Let $\omega_{P_{\mathrm{o}_{s}}, 2}^{(2)}(P), s=1,2,3$, denote the normalized Abelian differential of the second kind satisfying

$$
\begin{gathered}
\int_{a_{k}} \omega_{P_{\infty_{s}}, 2}^{(2)}(P)=0, \quad k=1, \cdots, m-2, \\
\omega_{P_{\infty_{s}}, 2}^{(2)}(P) \underset{\zeta \rightarrow 0}{=}\left(\zeta^{-2}+O(1)\right) d \zeta \text { as } P \rightarrow P_{\infty_{s}},
\end{gathered}
$$

and introduce

$$
\Omega^{(2)}(P)=-i \omega_{P_{\mathrm{o}_{1}}, 2}^{(2)}(P)+2 i \omega_{P_{\mathrm{oO}_{2}}, 2}^{(2)}(P)+i \omega_{P_{\mathrm{o}_{3}}, 2}^{(2)}(P),
$$

then we have

$$
\int_{Q_{0}}^{P} \Omega^{(2)}(P)= \begin{cases}i \zeta^{-1}+e_{1}^{(2)}\left(Q_{0}\right)+O(\zeta), & \text { as } P \rightarrow P_{\infty_{1}} \\ -2 i \zeta^{-1}+e_{2}^{(2)}\left(Q_{0}\right)+O(\zeta), & \text { as } P \rightarrow P_{\infty_{2}} \\ -i \zeta^{-1}+e_{3}^{(2)}\left(Q_{0}\right)+O(\zeta), & \text { as } P \rightarrow P_{\infty_{3}}\end{cases}
$$

where $e_{1}^{(2)}\left(Q_{0}\right), e_{2}^{(2)}\left(Q_{0}\right), e_{3}^{(2)}\left(Q_{0}\right)$ are integral constants and $Q_{0}$ is an appropriately chosen base point on $\mathscr{K}_{m-2} \backslash\left\{P_{\infty_{1}}\right.$, $\left.P_{\infty_{2}}, P_{\infty_{3}}\right\}$. The $b$-periods of the differential $\Omega^{(2)}(P)$ are denoted by

$$
\begin{aligned}
U_{2}^{(2)} & =\left(U_{2,1}^{(2)}, \cdots, U_{2, m-2}^{(2)}\right), \\
U_{2, k}^{(2)} & =\frac{1}{2 \pi i} \int_{b_{k}} \Omega^{(2)}(P), \\
k & =1, \cdots, m-2 .
\end{aligned}
$$


Then, from (85) and (87), we have

$$
\begin{aligned}
U_{2, k}^{(2)}= & \frac{1}{2 \pi i}\left[-i \int_{b_{k}} \omega_{P_{\infty_{1}, 2}}^{(2)}(P)+2 i \int_{b_{k}} \omega_{P_{\infty_{2}}, 2}^{(2)}(P)+i \int_{b_{k}} \omega_{P_{\infty_{3}, 2}}^{(2)}(P)\right] \\
= & -i \frac{C_{k, 2 n-1}+\alpha_{0} C_{k, 3 n-2}}{\left(\alpha_{0}-\beta_{0}\right)\left(2 \alpha_{0}+\beta_{0}\right)}+2 i \frac{C_{k, 2 n-1}+\beta_{0} C_{k, 3 n-2}}{\left(\alpha_{0}-\beta_{0}\right)\left(\alpha_{0}+2 \beta_{0}\right)} \\
& -i \frac{C_{k, 2 n-1}-\left(\alpha_{0}+\beta_{0}\right) C_{k, 3 n-2}}{\left(\alpha_{0}+2 \beta_{0}\right)\left(2 \alpha_{0}+\beta_{0}\right)} \\
= & \frac{1}{\left(\alpha_{0}+2 \beta_{0}\right)\left(2 \alpha_{0}+\beta_{0}\right)\left(\alpha_{0}-\beta_{0}\right)} \\
& \cdot\left[\left(4 i \alpha_{0}+5 i \beta_{0}\right) C_{k, 2 n-1}+\left(2 i \alpha_{0}^{2}+6 i \alpha_{0} \beta_{0}+i \beta_{0}^{2}\right) C_{k, 3 n-2}\right],
\end{aligned}
$$

in which we used $1 / 2 \pi i \int_{b_{k}} \omega_{Q_{1}, 2}^{(2)}(P)=\rho_{k, 0}$, if $w_{k}=\sum_{l=0}^{\infty} \rho_{k, l} \zeta_{Q_{1}}$ $d \zeta_{Q_{1}}$

Furthermore, the normalized Abelian differential of the third kind $\omega_{P_{\infty_{3}}, P_{\infty_{j}}}^{(3)}(P), j=1,2$, is holomorphic on $\mathscr{K}_{m-2} \backslash\{$ $\left.P_{\mathrm{\infty}_{3}}, P_{\mathrm{\infty}_{j}}\right\}$ with simple poles at $P_{\mathrm{\infty}_{3}}$ and $P_{\mathrm{\infty}_{j}}$ with residues 1 and -1 , respectively, that is,

$$
\begin{aligned}
& \omega_{P_{\infty_{3}}, P_{\infty_{1}}}^{(3)}(P) \underset{\zeta \rightarrow 0}{=} \begin{cases}\left(-\zeta^{-1}+O(1)\right) d \zeta, & \text { as } P \rightarrow P_{\infty_{1}}, \\
O(1) d \zeta, & \text { as } P \rightarrow P_{\infty_{2}}, \\
\left(\zeta^{-1}+O(1)\right) d \zeta, & \text { as } P \rightarrow P_{\infty_{3}},\end{cases} \\
& \omega_{P_{\mathrm{\infty}_{3}}, P_{\mathrm{\infty}_{2}}}^{(3)}(P) \underset{\zeta \rightarrow 0}{=} \begin{cases}O(1) d \zeta, & \text { as } P \rightarrow P_{\mathrm{\infty}_{1}}, \\
\left(-\zeta^{-1}+O(1)\right) d \zeta, & \text { as } P \rightarrow P_{\mathrm{\infty}_{2}}, \\
\left(\zeta^{-1}+O(1)\right) d \zeta, & \text { as } P \rightarrow P_{\mathrm{\infty}_{3}},\end{cases} \\
& \int_{Q_{0}}^{P} \omega_{P_{\infty_{3}}, P_{\infty_{1}}}^{(3)}(P)= \begin{cases}-\ln \zeta+e_{1, \infty_{1}}^{(3)}\left(Q_{0}\right)+O(\zeta), & \text { as } P \longrightarrow P_{\infty_{1}}, \\
e_{1, \infty_{2}}^{(3)}\left(Q_{0}\right)+O(\zeta), & \text { as } P \longrightarrow P_{\infty_{2}}, \\
\ln \zeta+e_{1, \infty_{3}}^{(3)}\left(Q_{0}\right)+O(\zeta), & \text { as } P \longrightarrow P_{\infty_{3}},\end{cases} \\
& \int_{Q_{0}}^{P} \omega_{P_{\mathrm{\infty}_{3}}, P_{\mathrm{\infty}_{2}}}^{(3)}(P)= \begin{cases}e_{2, \infty_{1}}^{(3)}\left(Q_{0}\right)+O(\zeta), & \text { as } P \longrightarrow P_{\mathrm{\infty}_{1}}, \\
-\ln \zeta+e_{2, \infty_{2}}^{(3)}\left(Q_{0}\right)+O(\zeta), & \text { as } P \longrightarrow P_{\mathrm{\infty}_{2}}, \\
\ln \zeta+e_{2, \infty_{3}}^{(3)}\left(Q_{0}\right)+O(\zeta), & \text { as } P \longrightarrow P_{\mathrm{\infty}_{3}},\end{cases}
\end{aligned}
$$

where $e_{k, \infty_{j}}^{(3)}\left(Q_{0}\right), k=1,2, j=1,2,3$, are integration constants. Let $\mathscr{T}_{m-2}$ be the period lattice $\left\{\underline{Z} \in \mathbb{C}^{m-2} \mid \underline{Z}=\underline{N}+\tau \underline{L} ; \underline{N}, \underline{L}\right.$ $\left.\in \mathbb{Z}^{m-2}\right\}$. The complex torus $\mathscr{J}_{m-2}=\mathbb{C}^{m-2} / \mathscr{T}_{m-2}$ is called the Jacobian variety of $\mathscr{K}_{m-2}$. An Abel map $\mathscr{A}: \mathscr{K}_{m-2} \longrightarrow$ $\mathcal{F}_{m-2}$ is defined by
$\mathscr{A}(P)=\left(\mathscr{A}_{1}(P), \cdots, \mathscr{A}_{m-2}(P)\right)=\left(\int_{Q_{0}}^{P} \omega_{1}, \cdots, \int_{Q_{0}}^{P} \omega_{m-2}\right)\left(\bmod \mathscr{T}_{m-2}\right)$,

with the natural linear extension to the factor group $\operatorname{Div}\left(\mathscr{K}_{m-2}\right)$

$$
\mathscr{A}\left(\sum n_{k} P_{k}\right)=\sum n_{k} \mathscr{A}\left(P_{k}\right)
$$

Considering the nonspecial divisor $\mathfrak{D}_{\widehat{\underline{\mu}}(x)}=\sum_{k-1}^{m-2} \widehat{\mu}_{k}(x)$, $\mathfrak{D}_{\underline{\underline{\hat{v}}}(x)}=\sum_{k-1}^{m-2} \widehat{\boldsymbol{v}}_{k}(x)$, and $\mathfrak{D}_{\widehat{\xi}_{(x)}}=\sum_{k-1}^{m-2} \widehat{\xi}_{k}(\bar{x})$, we define

$$
\mathscr{A}\left(\sum_{k=1}^{m-2} P_{k}^{(j)}(x)\right)=\sum_{k=1}^{m-2} \mathscr{A}\left(P_{k}^{(j)}(x)\right)=\sum_{k=1}^{m-2} \int_{Q_{0}}^{P_{k}^{(j)}(x)} \omega=\rho^{(j)}(x), \quad j=1,2,3,
$$

where $P_{k}^{(1)}(x)=\widehat{\mu}_{k}(x), P_{k}^{(2)}(x)=\widehat{v}_{k}(x), P_{k}^{(3)}(x)=\widehat{\xi}_{k}(x), \widehat{\mu}(x)$ $=\left(\widehat{\mu}_{1}(x), \cdots, \widehat{\mu}_{m-2}(x)\right) \in \sigma^{m-2} \mathscr{K}_{m-2}, \underline{\widehat{v}}(x)=\left(\widehat{v}_{1}(x), \cdots\right.$,

$\left.\widehat{v}_{m-2}(x)\right) \in \sigma^{m-2} \mathscr{K}_{m-2}, \underline{\hat{\xi}}(x)=\left(\widehat{\xi}_{1}(x), \cdots, \widehat{\xi}_{m-2}(x)\right) \in \sigma^{m-2}$ $\mathscr{K}_{m-2}$, and

$$
\sigma^{m-2} \mathscr{K}_{m-2}=\left\{\left\{Q_{1}, \cdots, Q_{n}\right\} \mid Q_{j} \in \mathscr{K}_{m-2}, j=1, \cdots, n\right\}
$$

denotes the $n$th symmetric power of $\mathscr{K}_{m-2}$.

Theorem 3. Assume that the curve $\mathscr{K}_{m-2}$ is nonsingular, and let $x, x_{0} \in \mathbb{C}$. Then,

$$
\begin{aligned}
& \rho^{(1)}(x)=\rho^{(1)}\left(x_{0}\right)+U_{2}^{(2)}\left(x-x_{0}\right), \\
& \rho^{(2)}(x)=\rho^{(2)}\left(x_{0}\right)+U_{2}^{(2)}\left(x-x_{0}\right), \\
& \rho^{(3)}(x)=\rho^{(3)}\left(x_{0}\right)+U_{2}^{(2)}\left(x-x_{0}\right) .
\end{aligned}
$$

Proof. We prove only the first linearity of the Abel map with respect to $x$ in (98). Assume that $\mu_{j}(x) \neq \mu_{j}^{\prime}(x)$ for $j \neq j^{\prime}$; then, one computes

$$
\begin{aligned}
& \frac{d}{d x} \rho_{l}^{(1)}(x)=\frac{d}{d x} \sum_{j=1}^{m-2} \int_{Q_{0}}^{\widehat{\mu}_{j}} \omega_{l}=\sum_{j=1}^{m-2} \mu_{j, x} \omega_{l}\left(\widehat{\mu}_{j}\right)=\sum_{j=1}^{m-2} \mu_{j,} \sum_{k=1}^{m-2} C_{l k} \bar{\omega}_{k} \\
& =\sum_{j=1}^{m-2} \frac{2\left[V_{12}^{(n)}\left(\mu_{j}, x\right)-u_{1} V_{13}^{(n)}\left(\mu_{j} x\right)\right]\left[3 y^{2}\left(\widehat{\mu}_{j}\right)+S_{m}\left(\mu_{j}\right)\right]}{\left(\alpha_{0}-\beta_{0}\right)\left(\alpha_{0}+2 \beta_{0}\right)\left(2 \alpha_{0}+\beta_{0}\right) u_{1} \prod_{k=1}^{m-2}\left(\mu_{j}\left(\mu_{j}(x)-\mu_{r}(x)\right)\right.} \\
& \cdot\left(\sum_{k=1}^{m-n-1} C_{l k} \frac{\mu_{j}^{k-1}}{3 y^{2}\left(\widehat{\mu}_{j}\right)+S_{m}\left(\mu_{j}\right)}+\sum_{k=m-n}^{m-2} C_{l k} \frac{y\left(\widehat{\mu}_{j}\right) \mu_{j}^{k+n-m}}{3 y^{2}\left(\widehat{\mu}_{j}\right)+S_{m}\left(\mu_{j}\right)}\right) \\
& =-\frac{2}{\left(\alpha_{0}-\beta_{0}\right)\left(\alpha_{0}+2 \beta_{0}\right)\left(2 \alpha_{0}+\beta_{0}\right)}\left\{\sum_{j=1}^{m-2 m} \sum_{k=1}^{m-n-1} C_{l k} \frac{\left[u_{1} V_{13}^{(n)}\left(\mu_{j}, x\right)-V_{12}^{(n)}\left(\mu_{j}, x\right)\right] \mu_{j}^{k-1}}{u_{1} \prod_{r=1}^{m-2}\left(\mu_{j}\left(\mu_{j}-\mu_{r}\right)\right.}\right. \\
& \left.+\sum_{j=1}^{m-2} \sum_{k=m-n}^{m-2} C_{l k} \frac{\left[u_{1} V_{13}^{(n)}\left(\mu_{j}, x\right)-V_{12}^{(n)}\left(\mu_{j}, x\right)\right] y\left(\widehat{\mu}_{j}\right) \mu_{j}^{k+n-m}}{u_{1} \prod_{r=1}^{m-2}\left(\mu_{j \neq j}\left(\mu_{j}-\mu_{r}\right)\right.}\right\} \\
& =-\frac{2}{\left(\alpha_{0}-\beta_{0}\right)\left(\alpha_{0}+2 \beta_{0}\right)\left(2 \alpha_{0}+\beta_{0}\right)}\left\{\sum_{k=1}^{m-n-1} C_{l k} \sum_{j=1}^{m-2} \frac{\left(-2 i \alpha_{0}-5 / 2 i \beta_{0}\right) \mu_{j}^{k+n-2}}{\prod_{r=1}^{m-2}\left(\mu_{j}\left(\mu_{j}-\mu_{r}\right)\right.}\right.
\end{aligned}
$$

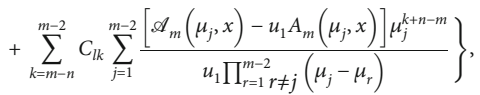


which yields by the use of the standard Lagrange interpolation argument that

$$
\frac{d}{d x} \rho_{l}^{(1)}(x)=U_{2, l}^{(2)}
$$

which implies the first representation of (98). The second and third equalities in (98) follow from the same calculation.

Denote by $\theta(\underline{z})$ the Riemann theta function associated with $\mathscr{K}_{m-2}$ equipped with a fixed homology basis. For convenience, the function $\underline{z}: \mathscr{K}_{m-2} \times \sigma^{m-2} \mathscr{K}_{m-2} \rightarrow \mathbb{C}$ is defined as

$$
\begin{aligned}
\underline{z}(P, \underline{Q}) & =M-\mathscr{A}(P)+\sum_{Q^{\prime} \underline{\underline{Q}}} \mathfrak{D}\left(Q^{\prime}\right) \mathscr{A}\left(Q^{\prime}\right), \quad P \in \mathscr{K}_{m-2}, \\
\underline{Q} & =\left(Q_{1}, \cdots, Q_{m-2}\right) \in \sigma^{m-2} \mathscr{K}_{m-2}, \quad j=1,2,3
\end{aligned}
$$

where $M$ is the vector of Riemann constants. Then, we get

$$
\begin{array}{ll}
\underline{z}(P, \underline{\widehat{\mu}}(x))=M-\mathscr{A}(P)+\rho^{(1)}(x), & P \in \mathscr{K}_{m-2}, \\
\underline{z}(P, \underline{\widehat{\hat{v}}}(x))=M-\mathscr{A}(P)+\rho^{(2)}(x), & P \in \mathscr{K}_{m-2}, \\
\underline{z}(P, \underline{\widehat{\xi}}(x))=M-\mathscr{A}(P)+\rho^{(3)}(x), & P \in \mathscr{K}_{m-2} .
\end{array}
$$

In view of (98), we could rewrite them as

$$
\begin{aligned}
& \underline{z}\left(P_{\infty_{j}}, \underline{\widehat{\mu}}(x)\right)=M_{1}^{(j)}+U_{2}^{(2)} x, \\
& \underline{z}\left(P_{\infty_{j}}, \underline{\widehat{v}}(x)\right)=M_{2}^{(j)}+U_{2}^{(2)} x, \\
& \underline{z}\left(P_{\infty_{j}}, \underline{\widehat{\xi}}(x)\right)=M_{3}^{(j)}+U_{2}^{(2)} x,
\end{aligned}
$$

where $M_{s}^{(j)}=M-\mathscr{A}\left(P_{\mathrm{O}_{j}}\right)+\rho^{(s)}\left(x_{0}\right)-U_{2}^{(2)} x_{0}, j=1,2,3, s=$ $1,2,3$.

Combined with the above results, the theta function representations of $\phi_{2}(P, x), \phi_{3}(P, x), \psi_{1}\left(P, x, x_{0}\right)$ and the algebro-geometric solutions of the stationary CCIRD hierarchy are presented in the next theorem.

Theorem 4. Assume that the curve $\mathscr{K}_{m-2}$ is nonsingular. Let $P=(\lambda, y) \in \mathscr{K}_{m-2} \backslash\left\{P_{\mathrm{O}_{1}}, P_{\mathrm{\infty}_{2}}, P_{\mathrm{\infty}_{3}}\right\}$ and let $\left(x, x_{0}\right) \in \mathbb{C}^{2}$. Suppose that $\mathfrak{D}_{\underline{\underline{\mu}}(x)}$ or $\mathfrak{D}_{\underline{\hat{v}}(x)}$ or $\mathfrak{D}_{\underline{\underline{\xi}}(x)}$ is nonspecial. Then,

$$
\begin{aligned}
\phi_{2}(P, x)= & N_{1}\left(x_{0}\right) \frac{\theta(\underline{z}(P, \underline{\hat{v}}(x))) \theta\left(\underline{z}\left(P_{\mathrm{\infty}_{3}}, \underline{\widehat{\mu}}(x)\right)\right)}{\theta\left(\underline{z}\left(P_{\mathrm{\infty}_{1}}, \widehat{\hat{v}}(x)\right)\right) \theta(\underline{z}(P, \underline{\hat{\mu}}(x)))} \exp \\
& \cdot\left(\int_{Q_{0}}^{P} \omega_{P_{\mathrm{\infty}_{3}}, P_{\infty_{1}}}^{(3)}+\left(e_{1}^{(2)}\left(Q_{0}\right)-e_{3}^{(2)}\left(Q_{0}\right)\right) x\right),
\end{aligned}
$$

$$
\begin{gathered}
\phi_{3}(P, x)=N_{2}\left(x_{0}\right) \frac{\theta(\underline{z}(P, \underline{\hat{\xi}}(x))) \theta\left(\underline{z}\left(P_{\infty_{3}}, \underline{\widehat{\mu}}(x)\right)\right)}{\theta\left(\underline{z}\left(P_{\infty_{2}}, \underline{\xi}(x)\right)\right) \theta(\underline{z}(P, \underline{\hat{\mu}}(x)))} \exp \\
\cdot\left(\int_{Q_{0}}^{P} \omega_{P_{\infty_{3}}, P_{\infty_{2}}}^{(3)}+\left(e_{2}^{(2)}\left(Q_{0}\right)-e_{3}^{(2)}\left(Q_{0}\right)\right) x\right), \\
\psi_{1}\left(P, x, x_{0}\right)=\frac{\theta(\underline{z}(P, \underline{\hat{\mu}}(x))) \theta\left(\underline{z}\left(P_{\infty_{3}}, \underline{\widehat{\mu}}\left(x_{0}\right)\right)\right)}{\theta\left(\underline{z}\left(P_{\infty_{3}}, \underline{\widehat{\mu}}(x)\right)\right) \theta\left(\underline{z}\left(P, \underline{\hat{\mu}}\left(x_{0}\right)\right)\right)} \exp \\
\cdot\left(\left(e_{3}^{(2)}\left(Q_{0}\right)-\int_{Q_{0}}^{P} \Omega^{(2)}\right)\left(x-x_{0}\right)\right),
\end{gathered}
$$

$$
\begin{aligned}
N_{1}\left(x_{0}\right)= & \frac{-2 i}{u_{1}\left(x_{0}\right)} \frac{\theta\left(\underline{z}\left(P_{\mathrm{\infty}_{1}}, \underline{\widehat{\mu}}\left(x_{0}\right)\right)\right)}{\theta\left(\underline{z}\left(P_{\mathrm{\infty}_{3}}, \underline{\hat{\mu}}\left(x_{0}\right)\right)\right)} \exp \\
& \cdot\left(\left(e_{3}^{(2)}\left(Q_{0}\right)-e_{1}^{(2)}\left(Q_{0}\right)\right) x_{0}-e_{1, \infty_{1}}^{(3)}\left(Q_{0}\right)\right), \\
N_{2}\left(x_{0}\right)= & i \frac{\theta\left(\underline{z}\left(P_{\infty_{2}}, \underline{\widehat{\mu}}\left(x_{0}\right)\right)\right)}{\theta\left(\underline{z}\left(P_{\infty_{3}}, \underline{\widehat{\mu}}\left(x_{0}\right)\right)\right)} \exp \\
& \cdot\left(\left(e_{3}^{(2)}\left(Q_{0}\right)-e_{2}^{(2)}\left(Q_{0}\right)\right) x_{0}-e_{2, \infty_{2}}^{(3)}\left(Q_{0}\right)\right) .
\end{aligned}
$$

Finally, the theta representations of $u_{1}(x)$ and $u_{2}(x)$ are of the form

$$
\begin{aligned}
& u_{1}(x)= u_{1}\left(x_{0}\right) \frac{\theta\left(\underline{z}\left(P_{\infty_{1}}, \underline{\widehat{\mu}}(x)\right)\right) \theta\left(\underline{z}\left(P_{\infty_{3}}, \underline{\widehat{\mu}}\left(x_{0}\right)\right)\right)}{\theta\left(\underline{z}\left(P_{\infty_{3}}, \underline{\widehat{\mu}}(x)\right)\right) \theta\left(\underline{z}\left(P_{\infty_{1}}, \underline{\widehat{\mu}}\left(x_{0}\right)\right)\right)} \exp \\
& \cdot\left(\left(e_{3}^{(2)}\left(Q_{0}\right)-e_{1}^{(2)}\left(Q_{0}\right)\right)\left(x-x_{0}\right)\right),
\end{aligned}
$$

$$
\begin{aligned}
u_{2}(x)= & \frac{4}{u_{1}\left(x_{0}\right)} \frac{\theta\left(\underline{z}\left(P_{\mathrm{\infty}_{3}}, \hat{\widehat{v}}(x)\right)\right) \theta\left(\underline{z}\left(P_{\mathrm{\infty}_{1}}, \underline{\widehat{\mu}}\left(x_{0}\right)\right)\right)}{\theta\left(\underline{z}\left(P_{\mathrm{\infty}_{1}}, \widehat{\hat{v}}(x)\right)\right) \theta\left(\underline{z}\left(P_{\mathrm{\infty}_{3}}, \underline{\widehat{\mu}}\left(x_{0}\right)\right)\right)} \\
& \times \exp \left(e_{1, \infty_{3}}^{(3)}\left(Q_{0}\right)-e_{1, \infty_{1}}^{(3)}\left(Q_{0}\right)\right. \\
& \left.-\left(e_{3}^{(2)}\left(Q_{0}\right)-e_{1}^{(2)}\left(Q_{0}\right)\right)\left(x-x_{0}\right)\right) .
\end{aligned}
$$

Proof. Let $\Psi_{1}$ be defined by the right-hand side of (106). We intend to prove that $\psi_{1}=\Psi_{1}$ with $\psi_{1}$ given by (64). For that purpose, we first inspect the zeros and poles of $\psi_{1}$. Since they can only come from zeros of $\phi_{2}$ and $\phi_{3}$, one can compute by using (34) and (35) that 


$$
\begin{aligned}
i \lambda+ & u_{1}(x) \phi_{2}(P, x)+\phi_{3}(P, x) \\
= & i \lambda+u_{1} \frac{y^{2} V_{13}^{(n)}-y A_{m}+B_{m}}{E_{m-2}}-\frac{y^{2} V_{12}^{(n)}-y \mathscr{A}_{m}+\mathscr{B}_{m}}{E_{m-2}} \\
= & \frac{1}{E_{m-2}}\left[\left(u_{1} V_{13}^{(n)}-V_{12}^{(n)}\right) y^{2}-\left(u_{1} A_{m}-\mathscr{A}_{m}\right) y\right. \\
& \left.+\frac{1}{3}\left(E_{m-2, x}-i \lambda E_{m-2}\right)+2\left(u_{1} V_{13}^{(n)}-V_{12}^{(n)}\right) S_{m}\right]+i \lambda \\
= & \frac{1}{3} \frac{E_{m-2, x}}{E_{m-2}}+\frac{2}{3} \frac{\left(u_{1} V_{13}^{(n)}-V_{12}^{(n)}\right)\left(3 y^{2}+S_{m}\right)}{E_{m-2}} \\
& -\frac{u_{1} V_{13}^{(n)} y\left(y+A_{m} / V_{13}^{(n)}\right)-V_{12}^{(n)} y\left(y+\mathscr{A}_{m} / V_{12}^{(n)}\right)}{E_{m-2}} \\
& +\frac{2}{3} i \lambda=-\frac{\mu_{j, x}(x)}{\lambda-\mu_{j}(x)}+O(1) \underset{\lambda \rightarrow \mu_{j}(x)}{=} \partial_{x} \ln \left(\lambda-\mu_{j}(x)\right)+O(1) .
\end{aligned}
$$

Then,

$$
\begin{aligned}
\psi_{1}\left(P, x, x_{0}\right)= & \exp \left(\int_{x_{0}}^{x}\left[i \lambda+u_{1}\left(x^{\prime}\right) \phi_{2}\left(P, x^{\prime}\right)+\phi_{3}\left(P, x^{\prime}\right)\right] d x^{\prime}\right) \\
& =\frac{\lambda-\mu_{j}(x)}{\lambda-\mu_{j}\left(x_{0}\right)} O(1)= \begin{cases}\left(\lambda-\mu_{j}(x)\right) O(1), & \text { for } P \text { near } \widehat{\mu}_{j}(x) \neq \widehat{\mu}_{j}\left(x_{0}\right), \\
O(1), & \text { for } P \text { near } \widehat{\mu}_{j}(x)=\widehat{\mu}_{j}\left(x_{0}\right), \\
\left(\lambda-\mu_{j}\left(x_{0}\right)\right)^{-1} O(1), & \text { for } P \text { near } \widehat{\mu}_{j}\left(x_{0}\right) \neq \widehat{\mu}_{j}(x),\end{cases}
\end{aligned}
$$

where $O(1) \neq 0$. Consequently, all zeros and poles of $\psi_{1}$ and $\Psi_{1}$ on $\mathscr{K}_{m-2} \backslash\left\{P_{\mathrm{\infty}_{1}}, P_{\mathrm{\infty}_{2}}, P_{\mathrm{\infty}_{3}}\right\}$ are simple and coincident. It remains to identify the essential singularities of $\psi_{1}$ and $\Psi_{1}$ at $P_{\infty_{1}}, P_{\mathrm{O}_{2}}, P_{\mathrm{\infty}_{3}}$. Considering (64), (76), (87), and the expression for $\Psi_{1}$ in (106), we deduce that $\psi_{1}$ and $\Psi_{1}$ share the same singularities and zeros. The Riemann-Roch uniqueness results in the holomorphic function $\Psi_{1} / \psi_{1}=\gamma$, where $\gamma$ is a constant. By (76), (87), and the right-hand side of (106), we have

$$
\begin{aligned}
\frac{\Psi_{1}\left(P, x, x_{0}\right)}{\psi_{1}\left(P, x, x_{0}\right)} \underset{\zeta \rightarrow 0}{=} \frac{\exp \left(i \zeta^{-1}\left(x-x_{0}\right)+O(\zeta)\right)(1+O(\zeta))}{\exp \left(i \zeta^{-1}\left(x-x_{0}\right)+O(\zeta)\right)} \\
\underset{\substack{\zeta \rightarrow 0 \\
=}}{1+O(\zeta), \quad P \rightarrow P_{\infty_{3}} .}
\end{aligned}
$$

Then, we conclude $\gamma=1$, with which the proof of (106) is completed. By using the asymptotic properties of $\psi_{1}$ near $P_{\mathrm{\infty}_{1}}$, we get (109). Equations (78), (79), and (93) immediately yield that $\phi_{2}$ and $\phi_{3}$ have the following forms:

$$
\begin{aligned}
& \phi_{2}(P, x)=\widehat{N}_{1}(x) \frac{\theta(\underline{z}(P, \underline{\widehat{v}}(x)))}{\theta(\underline{z}(P, \underline{\hat{\mu}}(x)))} \exp \left(\int_{Q_{0}}^{P} \omega_{P_{\infty_{3}}, P_{\infty_{1}}}^{(3)}\right), \\
& \phi_{3}(P, x)=\widehat{N}_{2}(x) \frac{\theta(\underline{z}(P, \underline{\hat{\xi}}(x)))}{\theta(\underline{z}(P, \underline{\hat{\mu}}(x)))} \exp \left(\int_{Q_{0}}^{P} \omega_{P_{\infty_{3}}, P_{\infty_{2}}}^{(3)}\right) .
\end{aligned}
$$

Taking into account the asymptotic expansions of $\phi_{2}$ and $\phi_{3}$ near $P_{\infty_{1}}, P_{\infty_{3}}$, we have

$$
\begin{aligned}
& u_{1}(x)=\frac{-2 i}{\widehat{N}_{1}(x)} \frac{\theta\left(\underline{z}\left(P_{\infty_{1}}, \underline{\widehat{\mu}}(x)\right)\right)}{\theta\left(\underline{z}\left(P_{\infty_{1}}, \underline{\widehat{v}}(x)\right)\right)} \exp \left(-e_{1, \infty_{1}}^{(3)}\left(Q_{0}\right)\right), \\
& u_{2}(x)=2 i \widehat{N}_{1}(x) \frac{\theta\left(\underline{z}\left(P_{\infty_{3}}, \underline{\widehat{v}}(x)\right)\right)}{\theta\left(\underline{z}\left(P_{\infty_{3}}, \underline{\widehat{\mu}}(x)\right)\right)} \exp \left(e_{1, \infty_{3}}^{(3)}\left(Q_{0}\right)\right), \\
& \frac{1}{u_{1}(x)}=\frac{3}{2} \widehat{N}_{2}(x) \frac{\theta\left(\underline{z}\left(P_{\infty_{1}}, \underline{\hat{\xi}}(x)\right)\right)}{\theta\left(\underline{z}\left(P_{\infty_{1}}, \underline{\widehat{\mu}}(x)\right)\right)} \exp \left(e_{2, \infty_{1}}^{(3)}\left(Q_{0}\right)\right),
\end{aligned}
$$

which together with (109) show the expressions (107), (108), and (110).

\section{Algebro-Geometric Solutions of the CCIRD Hierarchy}

In this section, we extend the results of Sections 3 and 4 to the time-dependent CCIRD hierarchy. In particular, we obtain Riemann theta function representations for the timedependent Baker-Akhiezer function, the meromorphic function, and algebro-geometric solutions of the CCIRD hierarchy.

Similar to (31)-(33), we consider the following timedependent Baker-Akhiezer function:

$$
\begin{aligned}
& \psi_{x}\left(P, x, x_{0}, t_{r}, t_{0, r}\right)=U\left(u\left(x, t_{r}\right) ; \lambda(P)\right) \psi\left(P, x, x_{0}, t_{r}, t_{0, r}\right), \\
& \psi_{t_{r}}\left(P, x, x_{0}, t_{r}, t_{0, r}\right)=\tilde{V}^{(r)}\left(u\left(x, t_{r}\right) ; \lambda(P)\right) \psi\left(P, x, x_{0}, t_{r}, t_{0, r}\right), \\
& V^{(n)}\left(u\left(x, t_{r}\right) ; \lambda(P)\right) \psi\left(P, x, x_{0}, t_{r}, t_{0, r}\right)=y(P) \psi\left(P, x, x_{0}, t_{r}, t_{0, r}\right), \\
& \psi_{1}\left(P, x_{0}, x_{0}, t_{0, r}, t_{0, r}\right)=1, \\
& P=(\lambda, y) \in \mathscr{K}_{m-2} \backslash\left\{P_{\infty_{1}}, P_{\infty_{2}}, P_{\infty_{3}}\right\}, x, t_{r}, x_{0}, t_{0, r} \in \mathbb{C} .
\end{aligned}
$$

The compatibility conditions of the first three equations in (116) show that

$$
\begin{gathered}
U_{t_{r}}-\tilde{V}_{x}^{(r)}+\left[U, V^{(r)}\right]=0, \\
-V_{x}^{(n)}+\left[U, V^{(n)}\right]=0, \\
-V_{t_{r}}^{(n)}+\left[\tilde{V}^{(r)}, V^{(n)}\right]=0 .
\end{gathered}
$$

It is easy to find that $y I-V^{(n)}$ satisfies (118) and (119). Then, the characteristic polynomial of Lax matrix $V^{(n)}$ for the CCIRD hierarchy is a constant independent of variables $x$ and $t_{r}$ with the expansion 


$$
\operatorname{det}\left(y I-V^{(n)}\right)=y^{3}+S_{m}(\lambda) y-T_{m}(\lambda)
$$

where $S_{m}(\lambda)$ and $T_{m}(\lambda)$ are defined as in (27) and (28). Then, the CCIRD curve $\mathscr{K}_{m-2}$ is determined by

$$
\mathscr{K}_{m-2}: \mathfrak{F}_{m}(\lambda, y)=y^{3}+S_{m}(\lambda) y-T_{m}(\lambda)=0
$$

Closely related to $\psi\left(P, x, x_{0}, t_{r}, t_{0, r}\right)$ are the following two meromorphic functions $\phi_{2}\left(P, x, t_{r}\right)$ and $\phi_{3}\left(P, x, t_{r}\right)$ on $\mathscr{K}_{m-2}$ defined by

$\phi_{2}\left(P, x, t_{r}\right)=\frac{\psi_{2}\left(P, x, x_{0}, t_{r}, t_{0, r}\right)}{\psi_{1}\left(P, x, x_{0}, t_{r}, t_{0, r}\right)}, P \in \mathscr{K}_{m-2}, x, t_{r}, x_{0}, t_{0, r} \in \mathbb{C}$,

$\phi_{3}\left(P, x, t_{r}\right)=\frac{\psi_{3}\left(P, x, x_{0}, t_{r}, t_{0, r}\right)}{\psi_{1}\left(P, x, x_{0}, t_{r}, t_{0, r}\right)}, P \in \mathscr{K}_{m-2}, x, t_{r}, x_{0}, t_{0, r} \in \mathbb{C}$,

which imply from (116) that

$$
\begin{aligned}
\phi_{2}\left(P, x, t_{r}\right) & =\frac{F_{m-2}\left(\lambda, x, t_{r}\right)}{y^{2} V_{23}^{(n)}\left(\lambda, x, t_{r}\right)-y C_{m}\left(\lambda, x, t_{r}\right)+D_{m}\left(\lambda, x, t_{r}\right)} \\
& =\frac{y^{2} V_{13}^{(n)}\left(\lambda, x, t_{r}\right)-y A_{m}\left(\lambda, x, t_{r}\right)+B_{m}\left(\lambda, x, t_{r}\right)}{E_{m-2}\left(\lambda, x, t_{r}\right)} \\
& =\frac{y V_{23}^{(n)}\left(\lambda, x, t_{r}\right)+C_{m}\left(\lambda, x, t_{r}\right)}{y V_{13}^{(n)}\left(\lambda, x, t_{r}\right)+A_{m}\left(\lambda, x, t_{r}\right)},
\end{aligned}
$$

$$
\begin{aligned}
\phi_{3}\left(P, x, t_{r}\right) & =\frac{\mathscr{F}_{m-2}\left(\lambda, x, t_{r}\right)}{y^{2} V_{32}^{(n)}\left(\lambda, x, t_{r}\right)-y \mathscr{C}_{m}\left(\lambda, x, t_{r}\right)+\mathscr{D}_{m}\left(\lambda, x, t_{r}\right)} \\
& =\frac{y^{2} V_{12}^{(n)}\left(\lambda, x, t_{r}\right)-y \mathscr{A}_{m}\left(\lambda, x, t_{r}\right)+\mathscr{B}_{m}\left(\lambda, x, t_{r}\right)}{-E_{m-2}\left(\lambda, x, t_{r}\right)} \\
& =\frac{y V_{32}^{(n)}\left(\lambda, x, t_{r}\right)+\mathscr{C}_{m}\left(\lambda, x, t_{r}\right)}{y V_{12}^{(n)}\left(\lambda, x, t_{r}\right)+\mathscr{A}_{m}\left(\lambda, x, t_{r}\right)},
\end{aligned}
$$

where $P=(\lambda, y) \in \mathscr{K}_{m-2},\left(x, t_{r}\right) \in \mathbb{C}^{2}$ and $A_{m}\left(\lambda, x, t_{r}\right), \cdots$, $D_{m}\left(\lambda, x, t_{r}\right), \mathscr{A}_{m}\left(\lambda, x, t_{r}\right), \cdots, \mathscr{D}_{m}\left(\lambda, x, t_{r}\right), E_{m-2}, F_{m-2}\left(\lambda, x, t_{r}\right.$ ), $\mathscr{F}_{m-2}\left(\lambda, x, t_{r}\right)$ are defined as in (36)-(38). Hence, (39)-(45) also hold in the present context. Similarly, we have

$$
\begin{aligned}
E_{m-2}\left(\lambda, x, t_{r}\right)= & \frac{\left(\alpha_{0}-\beta_{0}\right)\left(\alpha_{0}+2 \beta_{0}\right)\left(2 \alpha_{0}+\beta_{0}\right)}{2} \\
& \cdot u_{1}\left(x, t_{r}\right) \prod_{j=1}^{m-2}\left(\lambda-\mu_{j}\left(x, t_{r}\right)\right),
\end{aligned}
$$

$$
\begin{aligned}
F_{m-2}\left(\lambda, x, t_{r}\right)= & \frac{\left(\alpha_{0}-\beta_{0}\right)\left(\alpha_{0}+2 \beta_{0}\right)\left(2 \alpha_{0}+\beta_{0}\right)}{6} \\
& \cdot u_{2}\left(x, t_{r}\right) \prod_{j=1}^{m-2}\left(\lambda-v_{j}\left(x, t_{r}\right)\right), \\
\mathscr{F}_{m-2}\left(\lambda, x, t_{r}\right)= & \frac{\left(\beta_{0}-\alpha_{0}\right)\left(\alpha_{0}+2 \beta_{0}\right)\left(2 \alpha_{0}+\beta_{0}\right)}{3} \\
& \cdot \prod_{j=1}^{m-2}\left(\lambda-\xi_{j}\left(x, t_{r}\right)\right) .
\end{aligned}
$$

After defining $\widehat{\mu}_{j}\left(x, t_{r}\right), \widehat{v}_{j}\left(x, t_{r}\right), \widehat{\xi}_{j}\left(x, t_{r}\right)$ as (46)-(48) by replacing $(x)$ with $\left(x, t_{r}\right)$, one infers from (74), (75), (124), and $(128)$ that the divisors $\left(\phi_{2}\left(P, x, t_{r}\right)\right)$ and $\left(\phi_{3}\left(P, x, t_{r}\right)\right)$ of $\phi_{2}\left(P, x, t_{r}\right)$ and $\phi_{3}\left(P, x, t_{r}\right)$ are as follows:

$$
\begin{aligned}
& \left(\phi_{2}\left(P, x, t_{r}\right)\right)=\mathfrak{D}_{P_{\infty 0_{3}}, \widehat{v}_{1}\left(x, t_{r}\right), \cdots, \widehat{v}_{m-2}\left(x, t_{r}\right)}(P)-\mathfrak{D}_{P_{\infty_{1}}, \widehat{\mu}_{1}\left(x, t_{r}\right), \cdots, \widehat{\mu}_{m-2}\left(x, t_{r}\right)}(P), \\
& \left(\phi_{3}\left(P, x, t_{r}\right)\right)=\mathfrak{D}_{P_{\infty_{3}}, \widehat{\xi}_{1}\left(x, t_{r}\right), \cdots, \widehat{\xi}_{m-2}\left(x, t_{r}\right)}(P)-\mathfrak{D}_{P_{\infty_{2}}, \widehat{\mu}_{1}\left(x, t_{r}\right), \cdots, \widehat{\mu}_{m-2}\left(x, t_{r}\right)}(P) .
\end{aligned}
$$

Differentiating (122) and (123) with respect to $t_{r}$ and using (116), we get

$$
\begin{aligned}
\phi_{2, t_{r}}= & \left(\frac{\psi_{2}}{\psi_{1}}\right)_{t_{r}}=\frac{\tilde{V}_{21}^{(r)} \psi_{1}+\tilde{V}_{22}^{(r)} \psi_{2}+\tilde{V}_{23}^{(r)} \psi_{3}}{\psi_{1}} \\
& -\phi_{2} \frac{\tilde{V}_{11}^{(r)} \psi_{1}+\tilde{V}_{12}^{(r)} \psi_{2}+\tilde{V}_{13}^{(r)} \psi_{3}}{\psi_{1}} \\
= & \tilde{V}_{21}^{(r)}+\left(\tilde{V}_{22}^{(r)}-\tilde{V}_{11}^{(r)}\right) \phi_{2}+\tilde{V}_{23}^{(r)} \phi_{3}-\tilde{V}_{12}^{(r)} \phi_{2}^{2}-\tilde{V}_{13}^{(r)} \phi_{2} \phi_{3}, \\
\phi_{3, t_{r}}= & \left(\frac{\psi_{3}}{\psi_{1}}\right)_{t_{r}}=\frac{\tilde{V}_{31}^{(r)} \psi_{1}+\tilde{V}_{32}^{(r)} \psi_{2}+\tilde{V}_{33}^{(r)} \psi_{3}}{\psi_{1}} \\
& -\phi_{3} \frac{\tilde{V}_{11}^{(r)} \psi_{1}+\tilde{V}_{12}^{(r)} \psi_{2}+\tilde{V}_{13}^{(r)} \psi_{3}}{\psi_{1}}=\tilde{V}_{31}^{(r)} \\
& +\left(\tilde{V}_{33}^{(r)}-\tilde{V}_{11}^{(r)}\right) \phi_{3}+\tilde{V}_{32}^{(r)} \phi_{2}-\tilde{V}_{13}^{(r)} \phi_{3}^{2}-\tilde{V}_{12}^{(r)} \phi_{2} \phi_{3} .
\end{aligned}
$$

Further properties of $\phi_{2}\left(P, x, t_{r}\right)$ and $\phi_{3}\left(P, x, t_{r}\right)$ can be presented, similar to (55)-(63), replacing $(x)$ with $\left(x, t_{r}\right)$, $(P, x)$ with $\left(P, x, t_{r}\right)$, etc. The four important ones of that are given as follows:

$$
\begin{aligned}
& \phi_{2, x}\left(P, x, t_{r}\right)+u_{1}\left(x, t_{r}\right) \phi_{2}^{2}\left(P, \mathrm{x}, t_{r}\right)+\phi_{2}\left(P, x, t_{r}\right) \phi_{3}\left(P, x, t_{r}\right) \\
& \quad+2 i \lambda \phi_{2}\left(P, x, t_{r}\right)-\phi_{3}\left(P, x, t_{r}\right)-u_{2}\left(x, t_{r}\right)=0, \\
& \phi_{3, x}\left(P, x, t_{r}\right)+\phi_{3}^{2}\left(P, x, t_{r}\right)+u_{1}\left(x, t_{r}\right) \phi_{2}\left(P, x, t_{r}\right) \phi_{3}\left(P, x, t_{r}\right) \\
& \quad-i \lambda \phi_{3}\left(P, x, t_{r}\right)-\phi_{2}\left(P, x, t_{r}\right)-1=0,
\end{aligned}
$$




$$
\begin{aligned}
\phi_{2, t_{r}}\left(P, x, t_{r}\right)= & \tilde{V}_{21}^{(r)}\left(x, t_{r}\right)+\left(\tilde{V}_{22}^{(r)}\left(x, t_{r}\right)-\tilde{V}_{11}^{(r)}\left(x, t_{r}\right)\right) \\
& \cdot \phi_{2}\left(P, x, t_{r}\right)+\tilde{V}_{23}^{(r)}\left(x, t_{r}\right) \phi_{3}\left(P, x, t_{r}\right) \\
& -\tilde{V}_{12}^{(r)}\left(x, t_{r}\right) \phi_{2}^{2}\left(P, x, t_{r}\right)-\tilde{V}_{13}^{(r)}\left(x, t_{r}\right) \\
& \cdot \phi_{2}\left(P, x, t_{r}\right) \phi_{3}\left(P, x, t_{r}\right), \\
\phi_{3, t_{r}}\left(P, x, t_{r}\right)= & \tilde{V}_{31}^{(r)}\left(x, t_{r}\right)+\left(\tilde{V}_{33}^{(r)}\left(x, t_{r}\right)-\tilde{V}_{11}^{(r)}\left(x, t_{r}\right)\right) \\
& \cdot \phi_{3}\left(P, x, t_{r}\right)+\tilde{V}_{32}^{(r)}\left(x, t_{r}\right) \phi_{2}\left(P, x, t_{r}\right) \\
& -\tilde{V}_{13}^{(r)}\left(x, t_{r}\right) \phi_{3}^{2}\left(P, x, t_{r}\right)-\tilde{V}_{12}^{(r)}\left(x, t_{r}\right) \\
& \cdot \phi_{2}\left(P, x, t_{r}\right) \phi_{3}\left(P, x, t_{r}\right) .
\end{aligned}
$$

Lemma 5. Assume (116) and (117) and let $\left(\lambda, x, t_{r}\right) \in \mathbb{C}^{3}$. Then,

$$
\begin{gathered}
E_{m-2, t_{r}}\left(\lambda, x, t_{r}\right)=E_{m-2, x}\left(\lambda, x, t_{r}\right) \frac{V_{13}^{(n)} \tilde{V}_{12}^{(r)}-V_{12}^{(n)} \tilde{V}_{13}^{(r)}}{u_{1} V_{13}^{(n)}-V_{12}^{(n)}} \\
+E_{m-2}\left[3\left(\tilde{V}_{11}^{(r)}-\frac{u_{1} \tilde{V}_{13}^{(r)}-\tilde{V}_{12}^{(r)}}{u_{1} V_{13}^{(n)}-V_{12}^{(n)}} V_{11}^{(n)}\right)-i \lambda \frac{V_{13}^{(n)} \tilde{V}_{12}^{(r)}-V_{12}^{(n)} \tilde{V}_{13}^{(r)}}{\left.u_{1} V_{13}^{(n)}-V_{12}^{(n)}\right],}\right. \\
F_{m-2, t_{r}}\left(\lambda, x, t_{r}\right)=F_{m-2, x}\left(\lambda, x, t_{r}\right) \frac{V_{21}^{(n)} \tilde{V}_{23}^{(r)}-V_{23}^{(n)} \tilde{V}_{21}^{(r)}}{V_{21}^{(n)}-u_{2} V_{23}^{(n)}}+F_{m-2}\left(\lambda, x, t_{r}\right) \\
\cdot\left[3\left(\tilde{V}_{22}^{(r)}-\frac{\tilde{V}_{21}^{(r)}-u_{2} \tilde{V}_{23}^{(r)}}{V_{21}^{(n)}-u_{2} V_{23}^{(n)}} V_{22}^{(n)}\right)+5 i \lambda \frac{V_{21}^{(n)} \tilde{V}_{23}^{(r)}-V_{23}^{(n)} \tilde{V}_{21}^{(r)}}{V_{21}^{(n)}-u_{2} V_{23}^{(n)}}\right], \\
\mathscr{F}_{m-2, t_{r}}\left(\lambda, x, t_{r}\right)=\mathscr{F}_{m-2, x}\left(\lambda, x, t_{r}\right) \frac{V_{31}^{(n)} \tilde{V}_{32}^{(r)}-V_{32}^{(n)} \tilde{V}_{31}^{(r)}}{V_{31}^{(n)}-V_{32}^{(n)}}+\mathscr{F}_{m-2}\left(\lambda, x, t_{r}\right) \\
\cdot\left[3\left(\tilde{V}_{33}^{(r)}-\frac{\tilde{V}_{31}^{(r)}-\tilde{V}_{32}^{(r)}}{V_{31}^{(n)}-V_{32}^{(n)}} V_{33}^{(n)}\right)-4 i \frac{V_{31}^{(n)} \tilde{V}_{32}^{(r)}-V_{32}^{(n)} \tilde{V}_{31}^{(r)}}{V_{31}^{(n)}-V_{32}^{(n)}}\right] .
\end{gathered}
$$

Proof. Differentiating (59) (by replacing $(x)$ with $\left(x, t_{r}\right)$ ) with respect to $t_{r}$, we get

$$
\begin{aligned}
&\left(\frac{E_{m-2, x}}{\left.E_{m-2}\right)_{t_{r}}=} \partial_{x} \partial_{t_{r}}\left(\ln E_{m-2}\right)\right. \\
&=\left[u_{1}\left(\phi_{2}+\phi_{2}^{*}+\phi_{2}^{* *}\right)+\phi_{3}+\phi_{3}^{*}+\phi_{3}^{* *}\right]_{r_{r}} \\
&=\left[u_{1, t_{r}}+u_{1}\left(\tilde{V}_{22}^{(r)}-\tilde{V}_{11}^{(r)}\right)+\tilde{V}_{32}^{(r)}\right]\left(\phi_{2}+\phi_{2}^{*}+\phi_{2}^{* *}\right) \\
&+\left(\tilde{V}_{33}^{(r)}-\tilde{V}_{11}^{(r)}+u_{1} \tilde{V}_{23}^{(r)}\right)\left(\phi_{3}+\phi_{3}^{*}+\phi_{3}^{* *}\right) \\
&-u_{1} \tilde{V}_{12}^{(r)}\left[\phi_{2}^{2}+\left(\phi_{2}^{*}\right)^{2}+\left(\phi_{2}^{* *}\right)^{2}\right] \\
&-\tilde{V}_{13}^{(r)}\left[\phi_{3}^{2}+\left(\phi_{3}^{*}\right)^{2}+\left(\phi_{3}^{* *}\right)^{2}\right]-\left(u_{1} \tilde{V}_{13}^{(r)}+\tilde{V}_{12}^{(r)}\right) \\
& \cdot\left(\phi_{2} \phi_{3}+\phi_{2}^{*} \phi_{3}^{*}+\phi_{2}^{* *} \phi_{3}^{* *}\right)+3\left(u_{1} \tilde{V}_{21}^{(r)}+\tilde{V}_{31}^{(r)}\right) \\
&= {\left[\tilde{V}_{12}^{(r)}\left(\phi_{2}+\phi_{2}^{*}+\phi_{2}^{* *}\right)+\tilde{V}_{13}^{(r)}\left(\phi_{3}+\phi_{3}^{*}+\phi_{3}^{* *}\right)+3 \tilde{V}_{11}^{(r)}\right]_{x} . }
\end{aligned}
$$

Without loss of generality, we take the integration constant as zero, then get

$$
\begin{aligned}
& \partial_{t_{r}}\left(\ln E_{m-2}\left(\lambda, x, t_{r}\right)\right)=\tilde{V}_{12}^{(r)}\left(\phi_{2}+\phi_{2}^{*}+\phi_{2}^{* *}\right)+\tilde{V}_{13}^{(r)}\left(\phi_{3}+\phi_{3}^{*}+\phi_{3}^{* *}\right) \\
& \quad+3 \tilde{V}_{11}^{(r)}=\left[\frac{E_{m-2, x}}{E_{m-2}}\left(\lambda, x, t_{r}\right)-i \lambda\right] \frac{V_{13}^{(n)} \tilde{V}_{12}^{(r)}-V_{12}^{(n)} \tilde{V}_{13}^{(r)}}{u_{1} V_{13}^{(n)}-V_{12}^{(n)}} \\
& \quad+3\left(\tilde{V}_{11}^{(r)}-\frac{u_{1} \tilde{V}_{13}^{(r)}-\tilde{V}_{12}^{(r)}}{u_{1} V_{13}^{(n)}-V_{12}^{(n)}} V_{11}^{(n)}\right),
\end{aligned}
$$

which implies equation (132). Differentiating (55) (by replacing $(x)$ with $\left.\left(x, t_{r}\right)\right)$ with respect to $t_{r}$ and using (55), (60), and (63), we can deduce

$$
\begin{aligned}
&-\left(\frac{F_{m-2}}{\left.E_{m-2}\right)_{t_{r}}=} \phi_{2} \phi_{2}^{*} \phi_{2}^{* *}\left(\frac{\phi_{2, t_{r}}}{\phi_{2}}+\frac{\phi_{2, t_{r}}^{*}}{\phi_{2}^{*}}+\frac{\phi_{2, t_{r}}^{* *}}{\phi_{2}^{* *}}\right)=\phi_{2} \phi_{2}^{*} \phi_{2}^{* *}\left[\tilde{V}_{21}^{(r)}\right.\right. \\
& \cdot\left(\frac{1}{\phi_{2}}+\frac{1}{\phi_{2}^{*}}+\frac{1}{\phi_{2}^{* *}}\right)+\tilde{V}_{23}^{(r)}\left(\frac{\phi_{3}}{\phi_{2}}+\frac{\phi_{3}^{*}}{\phi_{2}^{*}}+\frac{\phi_{3}^{* *}}{\phi_{2}^{* *}}\right) \\
&+3 \tilde{V}_{22}^{(r)}=-\tilde{V}_{12}^{(r)}\left(\phi_{2}+\phi_{2}^{*}+\phi_{2}^{* *}\right) \\
&\left.-\tilde{V}_{13}^{(r)}\left(\phi_{3}+\phi_{3}^{*}+\phi_{3}^{* *}\right)-3 \tilde{V}_{11}^{(r)}\right] \\
&=-\frac{F_{m-2}}{E_{m-2}}\left[\frac{V_{21}^{(n)} \tilde{V}_{23}^{(r)}-V_{23}^{(n)} \tilde{V}_{21}^{(r)}}{V_{21}^{(n)}-u_{2} V_{23}^{(n)}}\left(\frac{F_{m-2, x}}{F_{m-2}}+5 i \lambda\right)\right. \\
&\left.+3\left(\tilde{V}_{22}^{(r)}-\frac{\tilde{V}_{21}^{(r)}-u_{2} \tilde{V}_{23}^{(r)}}{V_{21}^{(n)}-u_{2} V_{23}^{(n)}} V_{22}^{(n)}\right)-\frac{E_{m-2, t_{r}}}{E_{m-2}}\right] .
\end{aligned}
$$

Thus, we prove the expression (133). The last one can be proved in the same way.

We present some properties of $\psi_{1}\left(P, x, x_{0}, t_{r}, t_{0, r}\right)$ as follows.

Lemma 6. Assume (116) and (122), $P=(\lambda, y) \in \mathscr{K}_{m-2} \backslash\left\{P_{\infty}\right\}$, and let $\left(\lambda, x, x_{0}, t_{r}, t_{0, r}\right) \in \mathbb{C}^{5}$. Then,

$$
\begin{gathered}
\psi_{1}\left(P, x, x_{0}, t_{r}, t_{0, r}\right)=\exp \left(\int _ { x _ { 0 } } ^ { x } \left[i \lambda+u_{1}\left(x^{\prime}, t_{r}\right) \phi_{2}\left(P, x^{\prime}, t_{r}\right)\right.\right. \\
\left.+\phi_{3}\left(P, x^{\prime}, t_{r}\right)\right] d x^{\prime}+\int_{t_{0, r}}^{t_{r}}\left[\tilde{V}_{11}^{(r)}\left(\lambda, x_{0}, t^{\prime}\right)+\tilde{V}_{12}^{(r)}\left(\lambda, x_{0}, t^{\prime}\right)\right. \\
\left.\left.\cdot \phi_{2}\left(P, x_{0}, t^{\prime}\right)+\tilde{V}_{13}^{(r)}\left(\lambda, x_{0}, t^{\prime}\right) \phi_{3}\left(P, x_{0}, t^{\prime}\right)\right] d t^{\prime}\right), \\
\psi_{1}\left(P, x, x_{0}, t_{r}, t_{0, r}\right) \psi_{1}\left(P^{*}, x, x_{0}, t_{r}, t_{0, r}\right) \psi_{1}\left(P^{* *}, x, x_{0}, t_{r}, t_{0, r}\right) \\
==\frac{E_{m-2}\left(\lambda, x, t_{r}\right)}{E_{m-2}\left(\lambda, x_{0}, t_{0, r}\right)} \exp \left(2 i \lambda\left(x-x_{0}\right)\right),
\end{gathered}
$$




$$
\begin{aligned}
& \psi_{1}\left(P, x, x_{0}, t_{r}, t_{0, r}\right)=\left[\frac{E_{m-2}\left(\lambda, x, t_{r}\right)}{E_{m-2}\left(\lambda, x_{0}, t_{0, r}\right)}\right]^{1 / 3} \times \exp \\
& \cdot\left(\int_{x_{0}}^{x}\left[\frac{2}{3} i \lambda+\frac{y^{2}\left(u_{1} V_{13}^{(n)}-V_{12}^{(n)}\right)-y\left(u_{1} A_{m}-\mathscr{A}_{m}\right)+2 / 3\left(u_{1} V_{13}^{(n)}-V_{12}^{(n)}\right) S_{m}}{E_{m-2}}\right]\right. \\
& \left.\cdot d x^{\prime}+\int_{t_{0, r}}^{t_{r}} \frac{y^{2}\left(u_{1} V_{13}^{(n)}-V_{12}^{(n)}\right)-y\left(u_{1} A_{m}-\mathscr{A}_{m}\right)+2 / 3\left(u_{1} V_{13}^{(n)}-V_{12}^{(n)}\right) S_{m}}{E_{m-2}} d t^{\prime}\right) .
\end{aligned}
$$

Similar to Lemma 1, the zeros $\left\{\mu_{j}(x)\right\}_{j=1}^{m-2},\left\{v_{j}(x)\right\}_{j=1}^{m-2}$, and $\left\{\xi_{j}(x)\right\}_{j=1}^{m-2}$ of $E_{m-2}(\lambda, x), \quad F_{m-2}(\lambda, x)$, and $\mathscr{F}_{m}(\lambda, x)$ are described in terms of Dubrovin-type equations as follows.

\section{Lemma 7.}

(i) Assume the zeros $\left\{\mu_{j}\left(x, t_{r}\right)\right\}_{j=1}^{m-2}$ of $E_{m-2}\left(\lambda, x, t_{r}\right)$ remain distinct for $\left(x, t_{r}\right) \in \Omega_{\mu}$, where $\Omega_{\mu} \in \mathbb{C}^{2}$ is open and connected. Then, $\left\{\mu_{j}\left(x, t_{r}\right)\right\}_{j=1}^{m-2}$ satisfy the system of differential equations

$$
\begin{aligned}
& \mu_{j, x}\left(x, t_{r}\right)=\frac{2\left[V_{12}^{(n)}\left(\mu_{j}\left(x, t_{r}\right), x, t_{r}\right)-u_{1}\left(x, t_{r}\right) V_{13}^{(n)}\left(\mu_{j}\left(x, t_{r}\right), x, t_{r}\right)\right]\left[3 y^{2}\left(\widehat{\mu}_{j}\left(x, t_{r}\right)\right)+S_{m}\left(\mu_{j}\left(x, t_{r}\right)\right)\right]}{\left(\alpha_{0}-\beta_{0}\right)\left(\alpha_{0}+2 \beta_{0}\right)\left(2 \alpha_{0}+\beta_{0}\right) u_{1}\left(x, t_{r}\right) \prod_{k=1}^{m-2} k \neq j\left(\mu_{j}\left(x, t_{r}\right)-\mu_{k}\left(x, t_{r}\right)\right)}, \quad 1 \leq j \leq m-2, \\
& \mu_{j, t_{r}}\left(x, t_{r}\right)=\frac{\left.2\left[\tilde{V}_{13}^{(r)} V_{12}^{(n)}-\tilde{V}_{12}^{(r)} V_{13}^{(n)}\right]\right|_{\lambda=\mu_{j}\left(x, t_{r}\right)}\left[3 y^{2}\left(\widehat{\mu}_{j}\left(x, t_{r}\right)\right)+S_{m}\left(\mu_{j}\left(x, t_{r}\right)\right)\right]}{\left(\alpha_{0}-\beta_{0}\right)\left(\alpha_{0}+2 \beta_{0}\right)\left(2 \alpha_{0}+\beta_{0}\right) u_{1}\left(x, t_{r}\right) \prod_{k=1 k \neq j}^{m-2}\left(\mu_{j}\left(x, t_{r}\right)-\mu_{k}\left(x, t_{r}\right)\right)}, \quad 1 \leq j \leq m-2
\end{aligned}
$$

(ii) Assume the zeros $\left\{v_{j}\left(x, t_{r}\right)\right\}_{j=1}^{m-2}$ of $F_{m-2}\left(\lambda, x, t_{r}\right)$ and connected. Then, $\left\{v_{j}\left(x, t_{r}\right)\right\}_{j=1}^{m-2}$ satisfy the system remain distinct for $\left(x, t_{r}\right) \in \Omega_{v}$, where $\Omega_{v} \in \mathbb{C}^{2}$ is open

$$
\begin{aligned}
& v_{j, x}\left(x, t_{r}\right)=\frac{6\left[V_{21}^{(n)}\left(v_{j}\left(x, t_{r}\right), x, t_{r}\right)-u_{2}\left(x, t_{r}\right) V_{23}^{(n)}\left(v_{j}\left(x, t_{r}\right), x, t_{r}\right)\right]\left[3 y^{2}\left(\widehat{v}_{j}\left(x, t_{r}\right)\right)+S_{m}\left(v_{j}\left(x, t_{r}\right)\right)\right]}{\left(\alpha_{0}-\beta_{0}\right)\left(\alpha_{0}+2 \beta_{0}\right)\left(2 \alpha_{0}+\beta_{0}\right) u_{2}\left(x, t_{r}\right) \prod_{k=1}^{m-2} k \neq j}, \quad 1 \leq j \leq m-2, \\
& v_{j, t_{r}}\left(x, t_{r}\right)=\frac{\left.6\left[\tilde{V}_{23}^{(r)} V_{21}^{(n)}-\tilde{V}_{21}^{(r)} V_{23}^{(n)}\right]\right|_{\lambda=v_{j}\left(x, t_{r}\right)}\left[3 y^{2}\left(\widehat{v}_{j}\left(x, t_{r}\right)\right)+S_{m}\left(v_{j}\left(x, t_{r}\right)\right)\right]}{\left(\alpha_{0}-\beta_{0}\right)\left(\alpha_{0}+2 \beta_{0}\right)\left(2 \alpha_{0}+\beta_{0}\right) u_{2}\left(x, t_{r}\right) \prod_{k=1 k \neq j}^{m-2}\left(v_{j}\left(x, t_{r}\right)-v_{k}\left(x, t_{r}\right)\right)}, \quad 1 \leq j \leq m-2
\end{aligned}
$$

(iii) Assume the zeros $\left\{\xi_{j}\left(x, t_{r}\right)\right\}_{j=1}^{m-2}$ of $\mathscr{F}_{m-2}\left(\lambda, x, t_{r}\right)$ open and connected. Then, $\left\{\xi_{j}\left(x, t_{r}\right)\right\}_{j=1}^{m-2}$ satisfy the system of differential equations remain distinct for $\left(x, t_{r}\right) \in \Omega_{\xi}$, where $\Omega_{\xi} \in \mathbb{C}^{2}$ is

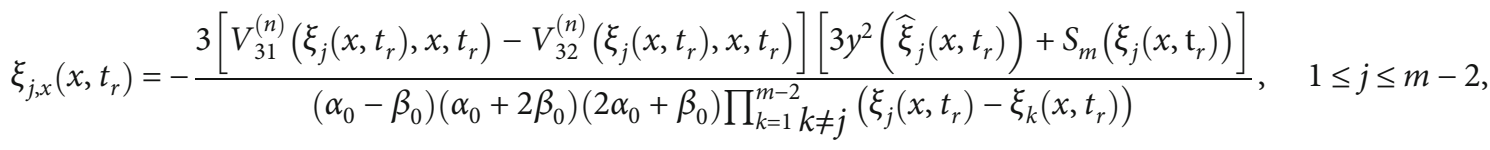

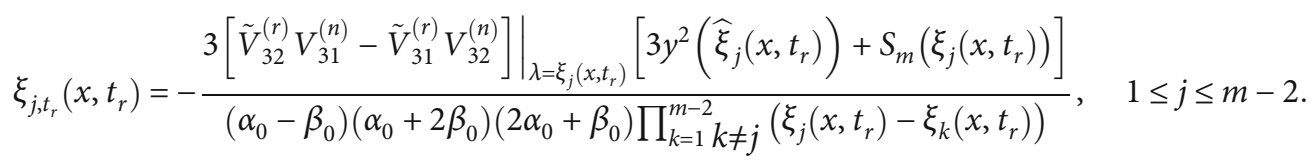


For convenience, we introduce the notation

$$
\tilde{V}_{1 j}^{(r)}= \begin{cases}\tilde{V}_{1 j}^{(r, 1,0)}=\sum_{k=0}^{r} \tilde{V}_{1 j, k-1}^{(1,0)} \lambda^{r-k}, & j=1,2,3, \\ \tilde{V}_{1 j}^{(r, 0,1)}=\sum_{k=0}^{r} \tilde{V}_{1 j, k-1}^{(0,1)} \lambda^{r-k}, & j=1,2,3,\end{cases}
$$

where

$$
\begin{aligned}
& \tilde{V}_{1 j, k-1}^{(1,0)}=\left.\tilde{V}_{1 j, k-1}\right|_{\tilde{\alpha}_{0}=1, \tilde{\beta}_{0}=0, \tilde{\alpha}_{1}, \cdots, \tilde{\alpha}_{r}, \tilde{\beta}_{1}, \cdots, \tilde{\beta}_{r} \in \mathbb{R}}, \quad j=1,2,3, \\
& \tilde{V}_{1 j, k-1}^{(0,1)}=\left.\tilde{V}_{1 j, k-1}\right|_{\tilde{\alpha}_{0}=0, \tilde{\beta}_{0}=1, \tilde{\alpha}_{1}, \cdots, \tilde{\alpha}_{r}, \tilde{\beta}_{1}, \cdots, \tilde{\beta}_{r} \in \mathbb{R}}, \quad j=1,2,3,
\end{aligned}
$$

and the corresponding homogeneous cases

$$
\overline{\tilde{V}}_{1 j}^{(r)}=\left\{\begin{array}{l}
\overline{\tilde{V}}_{1 j}^{(r, 1,0)}=\sum_{k=0}^{r} \overline{\tilde{V}}_{1 j, k-1}^{(1,0)} \lambda^{r-k}, \quad j=1,2,3, \\
\overline{\tilde{V}}_{1 j}^{(r, 0,1)}=\sum_{k=0}^{r} \overline{\tilde{V}}_{1 j, k-1}^{(0,1)} \lambda^{r-k}, \quad j=1,2,3,
\end{array}\right.
$$

with

$$
\begin{aligned}
& \overline{\tilde{V}}_{1 j, k-1}^{(1,0)}=\left.\tilde{V}_{1 j, k-1}\right|_{\tilde{\alpha}_{0}=1, \tilde{\beta}_{0}=0, \tilde{\alpha}_{1}=\cdots=\tilde{\alpha}_{r}=\tilde{\beta}_{1}=\cdots=\tilde{\beta}_{r}=0}, j=1,2,3, \\
& \overline{\tilde{V}}_{1 j, k-1}^{(0,1)}=\left.\tilde{V}_{1 j, k-1}\right|_{\tilde{\alpha}_{0}=0, \tilde{\beta}_{0}=1, \tilde{\alpha}_{1}=\cdots=\tilde{\alpha}_{r}=\tilde{\beta}_{1}=\cdots=\tilde{\beta}_{r}=0}, j=1,2,3 .
\end{aligned}
$$

In view of (138), we denote the function $I_{r}\left(p, x, t_{r}\right)$ by

$$
\begin{aligned}
I_{r}\left(P, x, t_{r}\right)= & \tilde{V}_{11}^{(r)}\left(\lambda, x, t_{r}\right)+\tilde{V}_{12}^{(r)}\left(\lambda, x, t_{r}\right) \phi_{2}\left(P, x, t_{r}\right) \\
& +\tilde{V}_{13}^{(r)}\left(\lambda, x, t_{r}\right) \phi_{3}\left(P, x, t_{r}\right),
\end{aligned}
$$

and $\bar{I}_{r}\left(p, x, t_{r}\right)$ is the associated homogeneous quantity replacing $\tilde{V}_{11}^{(r)}, \tilde{V}_{12}^{(r)}, \tilde{V}_{13}^{(r)}$ by the corresponding homogeneous polynomials $\overline{\tilde{V}}_{11}^{(r)}, \overline{\tilde{V}}_{12}^{(r)}, \overline{\tilde{V}}_{13}^{(r)}$, that is,

$$
\begin{aligned}
\bar{I}_{r}\left(P, x, t_{r}\right)= & \overline{\tilde{V}}_{11}^{(r)}\left(\lambda, x, t_{r}\right)+\overline{\tilde{V}}_{12}^{(r)}\left(\lambda, x, t_{r}\right) \phi_{2}\left(P, x, t_{r}\right) \\
& +\overline{\tilde{V}}_{13}^{(r)}\left(\lambda, x, t_{r}\right) \phi_{3}\left(P, x, t_{r}\right)
\end{aligned}
$$

especially

$$
\begin{aligned}
& \bar{I}_{r}^{(1,0)}=\left.\left(\tilde{V}_{11}^{(r)}+\tilde{V}_{12}^{(r)} \phi_{2}+\tilde{V}_{13}^{(r)} \phi_{3}\right)\right|_{\tilde{\alpha}_{0}=1, \tilde{\beta}_{0}=0, \tilde{\alpha}_{1}=\cdots=\tilde{\alpha}_{r}=\tilde{\beta}_{1}=\cdots=\tilde{\beta}_{r}=0}, \\
& \bar{I}_{r}^{(0,1)}=\left.\left(\tilde{V}_{11}^{(r)}+\tilde{V}_{12}^{(r)} \phi_{2}+\tilde{V}_{13}^{(r)} \phi_{3}\right)\right|_{\tilde{\alpha}_{0}=0, \tilde{\beta}_{0}=1, \tilde{\alpha}_{1}=\cdots=\tilde{\alpha}_{r}=\tilde{\beta}_{1}=\cdots=\tilde{\beta}_{r}=0} .
\end{aligned}
$$

Lemma 8. Assume $\left(x, t_{r}\right) \in \mathbb{C}^{2}, \lambda=\zeta^{-1}$ denotes the local coordinate near $P_{\infty_{j}}, j=1,2,3$. Then,

$$
\begin{gathered}
\bar{I}_{r}^{(1,0)}= \begin{cases}\zeta^{-r}+\frac{2 i}{u_{1}} \overline{\tilde{V}}_{12, r}^{(1,0)}+O(\zeta), & P \longrightarrow P_{\infty_{1}}, \\
-i \overline{\tilde{V}}_{13, r}^{(1,0)}+O(\zeta), & P \longrightarrow P_{\infty_{2}}, \\
\zeta^{-r}+O(\zeta), & P \longrightarrow P_{\infty_{3}},\end{cases} \\
\bar{I}_{r}^{(0,1)}= \begin{cases}\frac{2 i}{u_{1}} \overline{\tilde{V}}_{12, r}^{(0,1)}+O(\zeta), & P \longrightarrow P_{\infty_{1}}, \\
\zeta^{-r}-i \overline{\tilde{V}}_{13, r}^{(0,1)}+O(\zeta), & P \longrightarrow P_{\infty_{2}}, \\
\zeta^{-r}+O(\zeta), & P \longrightarrow P_{\infty_{3}} .\end{cases}
\end{gathered}
$$

Proof. We only prove (151) and accordingly obtain (152). From (149), it is easy to see that

$$
\begin{aligned}
\bar{I}_{r}^{(1,0)}= & -\zeta^{-1}+i u_{1} \phi_{2}-i \phi_{3} \\
& = \begin{cases}\zeta^{-1}+\frac{2 i}{u_{1}} \overline{\tilde{V}}_{12,1}^{(1,0)}+O(\zeta), & P \longrightarrow P_{\infty_{1}}, \\
-i \overline{\tilde{V}}_{13,1}^{(1,0)}+O(\zeta), & P \longrightarrow P_{\infty_{2}}, \\
\zeta^{-1}+O(\zeta), & P \longrightarrow P_{\infty_{3}} ;\end{cases}
\end{aligned}
$$

thus, (151) is right for $r=1$. By using (116), (117), and (149), we have

$$
\begin{aligned}
\partial_{x} \bar{I}_{r}^{(1,0)}\left(P, x, t_{r}\right)= & \partial_{x}\left[\overline{\tilde{V}}_{11}^{(r, 1,0)}\left(\lambda, x, t_{r}\right)+\overline{\tilde{V}}_{12}^{(r, 1,0)}\left(\lambda, x, t_{r}\right)\right. \\
& \left.\cdot \phi_{2}\left(P, x, t_{r}\right)+\overline{\tilde{V}}_{13}^{(r, 1,0)}\left(\lambda, x, t_{r}\right) \phi_{3}\left(P, x, t_{r}\right)\right] \\
= & \partial_{t}\left[i \lambda+u_{1}\left(x, t_{r}\right) \phi_{2}\left(P, x, t_{r}\right)+\phi_{3}\left(P, x, t_{r}\right)\right] .
\end{aligned}
$$

(i) When $P \rightarrow P_{\infty_{1}}$, by investigating (153), one can assume that $\bar{I}_{r}^{(1,0)}\left(P, x, t_{r}\right)$ has the following expansion

$$
\bar{I}_{r}^{(1,0)}\left(P, x, t_{r}\right) \underset{\zeta \rightarrow 0}{=\zeta^{-r}}+\sum_{j=0}^{\infty} \varsigma_{1 j}\left(x, t_{r}\right) \zeta^{j}, \text { as } P \rightarrow P_{\infty_{1}} \text {, }
$$

for some coefficients $\left\{\varsigma_{1 j}\left(x, t_{r}\right)\right\}_{j \in \mathbb{N}_{0}}$. Suppose that (as $P \longrightarrow$ $\left.P_{\infty_{1}}\right)$

$$
\begin{gathered}
\phi_{2}=\sum_{j=0}^{\infty} \kappa_{1, j-1} \zeta^{j-1}, \\
\phi_{3}=\sum_{j=0}^{\infty} \chi_{1, j} \zeta^{j},
\end{gathered}
$$


where $\kappa_{1, j-1}$ and $\chi_{1, j}$ are defined in (74) and (75). Substituting (155) and (156) into (154) and comparing the same powers of $\zeta$ yield

$$
\begin{gathered}
\varsigma_{1 j, x}=\left(u_{1} \kappa_{1, j}+\chi_{1, j}\right)_{t_{r}}, j=0,1,2, \cdots, \\
\varsigma_{10, x}=\left(u_{1} \kappa_{1,0}+\chi_{1,0}\right)_{t_{r}}=\left[\frac{2 i}{u_{1}} \overline{\tilde{V}}_{12, r}^{(1,0)}\right]_{x}, \\
\varsigma_{11, x}=\left(u_{1} \kappa_{1,1}+\chi_{1,1}\right)_{t_{r}}=\left[-\overline{\tilde{V}}_{11, r}^{(1,0)}-\frac{u_{1, x}}{u_{1}^{2}} \overline{\tilde{V}}_{12, r}^{(1,0)}\right. \\
\left.-\frac{2}{3 u_{1}}\left(\overline{\tilde{V}}_{13, r}^{(1,0)}-\frac{1}{u_{1}} \overline{\tilde{V}}_{12, r}^{(1,0)}\right)+\frac{2 i}{u_{1}} \overline{\tilde{V}}_{12, r+1}^{(1,0)}\right]_{x},
\end{gathered}
$$

from which it can be inferred

$$
\begin{gathered}
\varsigma_{10}\left(x, t_{r}\right)=\varepsilon_{1,0}\left(t_{r}\right)+\frac{2 i}{u_{1}} \overline{\tilde{V}}_{12, r}^{(1,0)}, \\
\varsigma_{11}\left(x, t_{r}\right)=\varepsilon_{1,1}\left(t_{r}\right)-\overline{\tilde{V}}_{11, r}^{(1,0)}-\frac{u_{1, x}}{u_{1}^{2}} \overline{\tilde{V}}_{12, r}^{(1,0)} \\
-\frac{2}{3 u_{1}}\left(\overline{\tilde{V}}_{13, r}^{(1,0)}-\frac{1}{u_{1}} \overline{\tilde{V}}_{12, r}^{(1,0)}\right)+\frac{2 i}{u_{1}} \overline{\tilde{V}}_{12, r+1}^{(1,0)},
\end{gathered}
$$

where $\varepsilon_{1,0}\left(t_{r}\right)$ and $\varepsilon_{1,1}\left(t_{r}\right)$ are integration constants. We find that the coefficients of the power series for $\phi_{2}\left(P, x, t_{r}\right), \phi_{3}$ $\left(P, x, t_{r}\right)$ near $P_{\mathrm{O}_{1}}$ and the coefficients of the homogeneous polynomials $\bar{V}_{1 j, r}^{(1,0)}$ are differential polynomials in $u$, with no arbitrary integration constants in their construction, and the definition of $\bar{I}_{r}^{(1,0)}$, it follows that it also can have no arbitrary integration constants and must consist purely of differential polynomials in $u$, and $\varepsilon_{1,0}\left(t_{r}\right)=\varepsilon_{1,1}\left(t_{r}\right)=0$. Therefore, we have

$$
\begin{aligned}
\bar{I}_{r}^{(1,0)}\left(P, x, t_{r}\right)= & \zeta^{-r}+\frac{2 i}{u_{1}} \overline{\tilde{V}}_{12, r}^{(1,0)}-\left[\overline{\tilde{V}}_{11, r}^{(1,0)}+\frac{u_{1, x}}{u_{1}^{2}} \overline{\tilde{V}}_{12, r}^{(1,0)}\right. \\
& \left.+\frac{2}{3 u_{1}}\left(\overline{\tilde{V}}_{13, r}^{(1,0)}-\frac{1}{u_{1}} \overline{\tilde{V}}_{12, r}^{(1,0)}\right)-\frac{2 i}{u_{1}} \overline{\tilde{V}}_{12, r+1}^{(1,0)}\right] \zeta \\
& +O\left(\zeta^{2}\right), \text { as } P \longrightarrow P_{\infty_{1}} .
\end{aligned}
$$

On the other hand, we find

$$
\begin{aligned}
\bar{I}_{r+1}^{(1,0)}\left(P, x, t_{r}\right) & =\overline{\tilde{V}}_{11}^{(r+1,1,0)}+\overline{\tilde{V}}_{12}^{(r+1,1,0)} \phi_{2}+\overline{\tilde{V}}_{13}^{(r+1,1,0)} \phi_{3} \\
& =\zeta^{-1} \bar{I}_{r}^{(1,0)}+\overline{\tilde{V}}_{11, r}^{(1,0)}+\overline{\tilde{V}}_{12, r}^{(1,0)} \phi_{2}+\overline{\tilde{V}}_{13, r}^{(1,0)} \phi_{3} \\
& =\zeta^{-r-1}+\frac{2 i}{u_{1}} \overline{\tilde{V}}_{12, r+1}^{(1,0)}+O(\zeta), \text { as } P \longrightarrow P_{\infty_{1}} .
\end{aligned}
$$

(ii) When $P \rightarrow P_{\mathrm{O}_{2}}$, by analysing (153), one can assume that $\bar{I}_{r}^{(1,0)}\left(P, x, t_{r}\right)$ has the following form:

$$
\bar{I}_{r}^{(1,0)}\left(P, x, t_{r}\right) \underset{\zeta \rightarrow 0}{=} \sum_{j=0}^{\infty} \varsigma_{2 j}\left(x, t_{r}\right) \zeta^{j}, \quad \text { as } P \rightarrow P_{\mathrm{\infty}_{2}},
$$

for some coefficients $\left\{\varsigma_{2 j}\left(x, t_{r}\right)\right\}_{j \in \mathbb{N}_{0}}$. Suppose that (as $P \rightarrow$ $\left.P_{\mathrm{\infty}_{2}}\right)$

$$
\begin{gathered}
\phi_{2}=\sum_{j=0}^{\infty} \kappa_{2, j} \zeta^{j}, \\
\phi_{3}=\sum_{j=0}^{\infty} \chi_{2, j-1} \zeta^{j-1},
\end{gathered}
$$

where $\kappa_{2, j}$ and $\chi_{2, j-1}$ are defined in (74) and (75). Inserting (161) and (162) into (154) and comparing the same powers of $\zeta$ imply

$$
\varsigma_{2 j, x}=\left(u_{1} \kappa_{2, j}+\chi_{2, j}\right)_{t_{r}}, j=0,1,2, \cdots
$$

$$
\begin{gathered}
\varsigma_{20, x}=\left(u_{1} \kappa_{2,0}+\chi_{2,0}\right)_{t_{r}}=\left[-i \overline{\tilde{V}}_{13, r}^{(1,0)}\right]_{x}, \\
\varsigma_{21, x}=\left(u_{1} \kappa_{2,1}+\chi_{2,1}\right)_{t_{r}} \\
=\left[-\overline{\tilde{V}}_{11, r}^{(1,0)}-\frac{1}{3}\left(\overline{\tilde{V}}_{12, r}^{(1,0)}-u_{1} \overline{\tilde{V}}_{13, r}^{(1,0)}\right)-i \overline{\tilde{V}}_{13, r+1}^{(1,0)}\right]_{x}
\end{gathered}
$$

from which it can be inferred

$$
\begin{gathered}
\varsigma_{20}\left(x, t_{r}\right)=\varepsilon_{2,0}\left(t_{r}\right)-i \overline{\tilde{V}}_{13, r}^{(1,0)}, \\
\varsigma_{21}\left(x, t_{r}\right)=\varepsilon_{2,1}\left(t_{r}\right)-\overline{\tilde{V}}_{11, r}^{(1,0)}-\frac{1}{3}\left(\overline{\tilde{V}}_{12, r}^{(1,0)}-u_{1} \overline{\tilde{V}}_{13, r}^{(1,0)}\right)-i \overline{\tilde{V}}_{13, r+1}^{(1,0)},
\end{gathered}
$$

where $\varepsilon_{2,0}\left(t_{r}\right)$ and $\varepsilon_{2,1}\left(t_{r}\right)$ are integration constants. Managed together, we find that $\varepsilon_{2,0}\left(t_{r}\right)=\varepsilon_{2,1}\left(t_{r}\right)=0$. Then,

$$
\begin{aligned}
\bar{I}_{r}^{(1,0)}\left(P, x, t_{r}\right)= & -i \overline{\tilde{V}}_{13, r}^{(1,0)}-\left[\overline{\tilde{V}}_{11, r}^{(1,0)}+\frac{1}{3}\left(\overline{\tilde{V}}_{12, r}^{(1,0)}-u_{1} \overline{\tilde{V}}_{12, r}^{(1,0)}\right)\right. \\
& \left.+i \overline{\tilde{V}}_{13, r+1}^{(1,0)}\right] \zeta+O\left(\zeta^{2}\right), \text { as } P \longrightarrow P_{\infty_{2}} .
\end{aligned}
$$


On the other hand, we get

$$
\begin{aligned}
\bar{I}_{r+1}^{(1,0)}\left(P, x, t_{r}\right) & =\overline{\tilde{V}}_{11}^{(r+1,1,0)}+\overline{\tilde{V}}_{12}^{(r+1,1,0)} \phi_{2}+\overline{\tilde{V}}_{13}^{(r+1,1,0)} \phi_{3} \\
& =\zeta^{-1} \bar{I}_{r}^{(1,0)}+\overline{\tilde{V}}_{11, r}^{(1,0)}+\overline{\tilde{V}}_{12, r}^{(1,0)} \phi_{2}+\overline{\tilde{V}}_{13, r}^{(1,0)} \phi_{3} \\
& =-i \overline{\tilde{V}}_{13, r+1}^{(1,0)}+O(\zeta), \text { as } P \longrightarrow P_{\mathrm{c}_{2}} .
\end{aligned}
$$

(iii) When $P \rightarrow P_{\mathrm{O}_{3}}$, in terms of (153), one can assume that $\bar{I}_{r}^{(1,0)}\left(P, x, t_{r}\right)$ has the following expansion:

$$
\bar{I}_{r}^{(1,0)}\left(P, x, t_{r}\right) \underset{\zeta \rightarrow 0}{=}-\zeta^{-r}+\sum_{j=1}^{\infty} \varsigma_{3 j}\left(x, t_{r}\right) \zeta^{j}, \quad \text { as } P \rightarrow P_{\mathrm{\infty}_{3}},
$$

for some coefficients $\left\{\varsigma_{3 j}\left(x, t_{r}\right)\right\}_{j \in \mathbb{N}}$. Suppose that (as $P \rightarrow$ $\left.P_{\mathrm{O}_{3}}\right)$

$$
\begin{aligned}
& \phi_{2}=\sum_{j=1}^{\infty} \kappa_{3, j} \zeta^{j}, \\
& \phi_{3}=\sum_{j=1}^{\infty} \chi_{3, j} \zeta^{j},
\end{aligned}
$$

where $\kappa_{3, j}$ and $\chi_{3, j}$ are defined in (74) and (75). Substituting (167) and (168) into (154) and comparing the same powers of $\zeta$ yield

$$
\begin{gathered}
\varsigma_{3 j, x}=\left(u_{1} \kappa_{3, j}+\chi_{3, j}\right)_{t_{r}}, j=0,1,2, \cdots, \\
\varsigma_{31, x}=\left(u_{1} \kappa_{3,1}+\chi_{3,1}\right)_{t_{r}}=\left[-\overline{\tilde{V}}_{11, r}^{(1,0)}\right]_{x},
\end{gathered}
$$

from which one can infer

$$
\varsigma_{31}\left(x, t_{r}\right)=\varepsilon_{3,1}\left(t_{r}\right)-\overline{\tilde{V}}_{11, r}^{(1,0)},
$$

where $\varepsilon_{3,1}\left(t_{r}\right)$ is an integration constant. Similarly, one can conclude $\varepsilon_{3,1}\left(t_{r}\right)=0$. Therefore,

$$
\bar{I}_{r}^{(1,0)}\left(P, x, t_{r}\right)=-\zeta^{-r}-\bar{V}_{11, r}^{(1,0)} \zeta+O\left(\zeta^{2}\right) \text {, as } P \longrightarrow P_{\infty_{3}} .
$$

On the other hand,

$$
\begin{aligned}
\bar{I}_{r+1}^{(1,0)}\left(P, x, t_{r}\right) & =\overline{\tilde{V}}_{11}^{(r+1,1,0)}+\overline{\tilde{V}}_{12}^{(r+1,1,0)} \phi_{2}+\overline{\tilde{V}}_{13}^{(r+1,1,0)} \phi_{3} \\
& =\zeta^{-1} \bar{I}_{r}^{(1,0)}+\overline{\tilde{V}}_{11, r}^{(1,0)}+\overline{\tilde{V}}_{12, r}^{(1,0)} \phi_{2}+\overline{\tilde{V}}_{13, r}^{(1,0)} \phi_{3} \\
& =-\zeta^{-r-1}+O(\zeta), \text { as } P \longrightarrow P_{\infty_{3}} .
\end{aligned}
$$

Thus, we complete the proof of (151). Similarly, we can prove that (152) is right.

From (116), one infers that

$$
I_{r}\left(P, x, t_{r}\right)=\sum_{l=0}^{r}\left(\tilde{\alpha}_{r-l} \bar{I}_{l}^{(1,0)}\left(P, x, t_{r}\right)+\tilde{\beta}_{r-l} \bar{I}_{l}^{(1,0)}\left(P, x, t_{r}\right) .\right.
$$

Therefore,

$$
\int_{t_{0, r}}^{t_{r}} I_{r}\left(P, x, t^{\prime}\right) d t^{\prime}= \begin{cases}\left(t_{r}-t_{0, r}\right) \sum_{l=0}^{r} \tilde{\alpha}_{r-l} \zeta^{-l}+\ln \frac{u_{1}\left(x, t_{r}\right)}{u_{1}\left(x, t_{0, r}\right)}+O(\zeta), & \text { as } P \rightarrow P_{\infty_{1},}, \\ \left(t_{r}-t_{0, r}\right) \sum_{l=0}^{r} \tilde{\beta}_{r-l} \zeta^{-l}+O(\zeta), & \text { as } P \rightarrow P_{\mathrm{oO}_{2},}, \\ \left(t_{0, r}-t_{r}\right) \sum_{l=0}^{r}\left(\tilde{\alpha}_{r-l}+\tilde{\beta}_{r-l}\right) \zeta^{-l}+O(\zeta), & \text { as } P \rightarrow P_{\mathrm{o}_{3}} .\end{cases}
$$

Let $\omega_{P_{\infty}, j}^{(2)}, j \in \mathbb{N}, l=1,2,3$, be the normalized differential of the second kind holomorphic on $\mathscr{K}_{m-2} \backslash\left\{P_{\infty_{l}}\right\}$ with a pole of order $j$ at $P_{\mathrm{o}_{l}}$,

$$
\omega_{P_{\infty_{l} j}^{j}}^{(2)}(P) \underset{\zeta \rightarrow 0}{=}\left(\zeta^{-j}+O(1)\right) d \zeta, \quad \text { as } P \rightarrow P_{\mathrm{\infty}_{l}} .
$$

Furthermore, we define the normalized differential of the second kind by

$$
\begin{aligned}
\tilde{\Omega}_{r+1}^{(2)}(P)= & \sum_{l=0}^{r} \tilde{\alpha}_{r-l} l \omega_{P_{\infty_{1}}, l+1}^{(2)}+\sum_{l=0}^{r} \tilde{\beta}_{r-l} l \omega_{P_{\infty_{2}}, l+1}^{(2)} \\
& -\sum_{l=0}^{r}\left(\tilde{\alpha}_{r-l}+\tilde{\beta}_{r-l}\right) l \omega_{P_{\infty_{3}}, l+1}^{(2)} .
\end{aligned}
$$

In addition, we define the vector of $b$-periods of the differential of the second kind $\tilde{\Omega}_{r+1}^{(2)}$,

$$
\begin{gathered}
\tilde{U}_{r+1}^{(2)}=\left(\tilde{U}_{r+1,1}^{(2)}, \cdots, \tilde{U}_{r+1, m-2}^{(2)}\right), \\
\tilde{U}_{r+1, k}^{(2)}=\frac{1}{2 \pi i} \int_{b_{k}} \tilde{\Omega}_{r+1}^{(2)}, \\
k=1, \cdots, m-2 .
\end{gathered}
$$

Integrating Equation (176) gives rise to

$$
\int_{Q_{0}}^{P} \tilde{\Omega}_{r+1}^{(2)}= \begin{cases}-\sum_{l=0}^{r} \tilde{\alpha}_{r-l} \zeta^{-l}+e_{1}^{(2)}\left(Q_{0}\right)+O(\zeta), & \text { as } P \rightarrow P_{\mathrm{\infty}_{1}}, \\ -\sum_{l=0}^{r} \tilde{\beta}_{r-l} \zeta^{-l}+e_{2}^{(2)}\left(Q_{0}\right)+O(\zeta), & \text { as } P \rightarrow P_{\mathrm{\infty}_{2}}, \\ \sum_{l=0}^{r}\left(\tilde{\alpha}_{r-l}+\tilde{\beta}_{r-l}\right) \zeta^{-l}+e_{3}^{(2)}\left(Q_{0}\right)+O(\zeta), & \text { as } P \rightarrow P_{\mathrm{\infty}_{3}},\end{cases}
$$


where $e_{1}^{(2)}\left(Q_{0}\right), e_{2}^{(2)}\left(Q_{0}\right), e_{3}^{(2)}\left(Q_{0}\right)$ are constants.

Given these results, the theta function representations of $\phi_{2}\left(P, x, t_{r}\right), \phi_{3}\left(P, x, t_{r}\right)$ and $\psi_{1}\left(P, x, x_{0}, t_{r}, t_{0, r}\right)$, particularly, the algebro-geometric solutions of CCIRD hierarchy, are shown as follows.

Theorem 9. Assume that the curve $\mathscr{K}_{m-2}$ is nonsingular. Let $P=(\lambda, y) \in \mathscr{K}_{m-2} \backslash\left\{P_{\infty_{1}}, P_{\mathrm{\infty}_{2}}, P_{\mathrm{\infty}_{3}}\right\}$ and let $\left(x, x_{0}, t_{r}, t_{0, r}\right) \in$ $\mathbb{C}^{4}$. Suppose that $\mathfrak{D}_{\underline{\underline{\mu}}\left(x, t_{r}\right)}$ or $\mathfrak{D}_{\underline{\underline{v}}\left(x, t_{r}\right)}$ or $\mathfrak{D}_{\underline{\underline{\xi}}\left(x, t_{r}\right)}$ is nonspecial. Then,

$$
\begin{gathered}
\phi_{2}\left(P, x, t_{r}\right)=N_{1}\left(x_{0}, t_{0, r}\right) \frac{\theta\left(\underline{z}\left(P, \underline{\hat{v}}\left(x, t_{r}\right)\right)\right) \theta\left(\underline{z}\left(P_{\infty_{3}}, \underline{\widehat{\mu}}\left(x, t_{r}\right)\right)\right)}{\theta\left(\underline{z}\left(P_{\infty_{1}}, \underline{\hat{v}}\left(x, t_{r}\right)\right)\right) \theta\left(\underline{z}\left(P, \underline{\hat{\mu}}\left(x, t_{r}\right)\right)\right)} \times \exp \\
\cdot\left(\int_{Q_{0}}^{P} \omega_{P_{\infty_{3}}, P_{\infty_{l}}}^{(3)}+\left(e_{1}^{(2)}\left(Q_{0}\right)-e_{3}^{(2)}\left(Q_{0}\right)\right) x+\left(e_{1}^{(2)}\left(Q_{0}\right)-e_{3}^{(2)}\left(Q_{0}\right)\right) t_{r}\right),
\end{gathered}
$$

$$
\begin{gathered}
\phi_{3}\left(P, x, t_{r}\right)=N_{2}\left(x_{0}, t_{0, r}\right) \frac{\theta\left(\underline{z}\left(P, \underline{\hat{\xi}}\left(x, t_{r}\right)\right)\right) \theta\left(\underline{z}\left(P_{\mathrm{O}_{3}}, \underline{\widehat{\mu}}\left(x, t_{r}\right)\right)\right)}{\theta\left(\underline{z}\left(P_{\mathrm{O}_{2}}, \widehat{\underline{\xi}}\left(x, t_{r}\right)\right)\right) \theta\left(\underline{z}\left(P, \underline{\hat{\mu}}\left(x, t_{r}\right)\right)\right)} \times \exp \\
\cdot\left(\int_{Q_{0}}^{P} \omega_{P_{\mathrm{O}_{3}}, P_{\infty_{2}}}^{(3)}+\left(e_{2}^{(2)}\left(Q_{0}\right)-e_{3}^{(2)}\left(Q_{0}\right)\right) x+\left(e_{2}^{(2)}\left(Q_{0}\right)-e_{3}^{(2)}\left(Q_{0}\right)\right) t_{r}\right),
\end{gathered}
$$

$$
\begin{aligned}
& \psi_{1}\left(P, x, x_{0}, t_{r}, t_{0, r}\right)=\frac{\theta\left(\underline{z}\left(P, \widehat{\underline{\mu}}\left(x, t_{r}\right)\right)\right) \theta\left(\underline{z}\left(P_{\infty_{3}}, \widehat{\underline{\mu}}\left(x_{0}, t_{0, r}\right)\right)\right)}{\theta\left(\underline{z}\left(P_{\infty_{3}}, \underline{\widehat{\mu}}\left(x, t_{r}\right)\right)\right) \theta\left(\underline{z}\left(P, \underline{\widehat{\mu}}\left(x_{0}, t_{0, r}\right)\right)\right)} \times \exp \\
& \cdot\left(\left(e_{3}^{(2)}\left(Q_{0}\right)-\int_{Q_{0}}^{P} \Omega^{(2)}\right)\left(x-x_{0}\right)+\left(e_{3}^{(2)}\left(Q_{0}\right)-\int_{Q_{0}}^{P} \tilde{\Omega}_{r+1}^{(2)}(P)\right)\left(t_{r}-t_{0, r}\right)\right),
\end{aligned}
$$

$$
\begin{aligned}
& N_{1}\left(x_{0}, t_{0, r}\right)=\frac{-2 i}{u_{1}\left(x_{0}, t_{0, r}\right)} \frac{\theta\left(\underline{z}\left(P_{\infty_{1}}, \underline{\widehat{\mu}}\left(x_{0}, t_{0, r}\right)\right)\right)}{\theta\left(\underline{z}\left(P_{\infty_{3}}, \underline{\widehat{\mu}}\left(x_{0}, t_{0, r}\right)\right)\right)} \times \exp \\
& \cdot\left(\left(e_{3}^{(2)}\left(Q_{0}\right)-e_{1}^{(2)}\left(Q_{0}\right)\right) x_{0}+\left(e_{3}^{(2)}\left(Q_{0}\right)-e_{1}^{(2)}\left(Q_{0}\right)\right) t_{0, r}-e_{1, \infty_{1}}^{(3)}\left(Q_{0}\right)\right),
\end{aligned}
$$

$$
\begin{aligned}
& N_{2}\left(x_{0}, t_{0, r}\right)=i \frac{\theta\left(\underline{z}\left(P_{\mathrm{O}_{2}}, \underline{\widehat{\mu}}\left(x_{0}, t_{0, r}\right)\right)\right)}{\theta\left(\underline{z}\left(P_{\mathrm{O}_{3}}, \underline{\widehat{\mu}}\left(x_{0}, t_{0, r}\right)\right)\right)} \times \exp \\
& \cdot\left(\left(e_{3}^{(2)}\left(Q_{0}\right)-e_{2}^{(2)}\left(Q_{0}\right)\right) x_{0}+\left(e_{3}^{(2)}\left(Q_{0}\right)-e_{2}^{(2)}\left(Q_{0}\right)\right) t_{0, r}-e_{2, O_{2}}^{(3)}\left(Q_{0}\right)\right) .
\end{aligned}
$$

Finally, the theta representations of $u_{1}\left(x, t_{r}\right)$ and $u_{2}\left(x, t_{r}\right)$ read

$$
\begin{gathered}
u_{1}\left(x, t_{r}\right)=u_{1}\left(x_{0}, t_{0, r}\right) \frac{\theta\left(\underline{z}\left(P_{\infty_{1}}, \underline{\widehat{\mu}}\left(x, t_{r}\right)\right)\right) \theta\left(\underline{z}\left(P_{\infty_{3}}, \underline{\widehat{\mu}}\left(x_{0}, t_{0, r}\right)\right)\right)}{\theta\left(\underline{z}\left(P_{\infty_{3}}, \underline{\widehat{\mu}}\left(x, t_{r}\right)\right)\right) \theta\left(\underline{z}\left(P_{\infty_{1}}, \underline{\widehat{\mu}}\left(x_{0}, t_{0, r}\right)\right)\right)} \times \exp \\
\cdot\left(\left(e_{3}^{(2)}\left(Q_{0}\right)-e_{1}^{(2)}\left(Q_{0}\right)\right)\left(x-x_{0}\right)+\left(e_{3}^{(2)}\left(Q_{0}\right)-e_{1}^{(2)}\left(Q_{0}\right)\right)\left(t_{r}-t_{0, r}\right)\right),
\end{gathered}
$$

$$
\begin{aligned}
& u_{2}\left(x, t_{r}\right)=\frac{4}{u_{1}\left(x_{0}, t_{0, r}\right)} \frac{\theta\left(\underline{z}\left(P_{\infty_{3}}, \underline{\widehat{v}}\left(x, t_{r}\right)\right)\right) \theta\left(\underline{z}\left(P_{\infty_{1}}, \underline{\widehat{\mu}}\left(x_{0}, t_{0, r}\right)\right)\right)}{\theta\left(\underline{z}\left(P_{\infty_{1}}, \widehat{\hat{v}}\left(x, t_{r}\right)\right)\right) \theta\left(\underline{z}\left(P_{\infty_{3}}, \underline{\widehat{\mu}}\left(x_{0}, t_{0, r}\right)\right)\right)} \times \exp \\
& \cdot\left(e_{1, \infty_{3}}^{(3)}\left(Q_{0}\right)-e_{1, \infty_{1}}^{(3)}\left(Q_{0}\right)-\left(e_{3}^{(2)}\left(Q_{0}\right)-e_{1}^{(2)}\left(Q_{0}\right)\right)\left(x-x_{0}\right)\right. \\
& \left.-\left(e_{3}^{(2)}\left(Q_{0}\right)-e_{1}^{(2)}\left(Q_{0}\right)\right)\left(t_{r}-t_{0, r}\right)\right) .
\end{aligned}
$$

Proof. The proof of (179), (180), and (182)-(185) is similar to that of Theorem 4 , so we only need to prove (181). Let $\Psi_{1}$ be defined by the right-hand side of (181). We want to prove that $\psi_{1}=\Psi_{1}$ with $\psi_{1}$ given by (138). Then, we compute by using (124) and (125) that

$$
\begin{aligned}
& i \lambda+u_{1}\left(x, t_{r}\right) \phi_{2}\left(P, x, t_{r}\right)+\phi_{3}\left(P, x, t_{r}\right) \\
& =i \lambda+u_{1} \frac{y^{2} V_{13}^{(n)}-y A_{m}+B_{m}}{E_{m-2}}-\frac{y^{2} V_{12}^{(n)}-y \mathscr{A}_{m}+\mathscr{B}_{m}}{E_{m-2}} \\
& =\frac{1}{E_{m-2}}\left[\left(u_{1} V_{13}^{(n)}-V_{12}^{(n)}\right) y^{2}-\left(u_{1} A_{m}-\mathscr{A}_{m}\right) y\right. \\
& \left.+\frac{1}{3}\left(E_{m-2, x}-i \lambda E_{m-2}\right)+2\left(u_{1} V_{13}^{(n)}-V_{12}^{(n)}\right) S_{m}\right]+i \lambda \\
& =\frac{1}{3} \frac{E_{m-2, x}}{E_{m-2}}+\frac{2}{3} \frac{\left(u_{1} V_{13}^{(n)}-V_{12}^{(n)}\right)\left(3 y^{2}+S_{m}\right)}{E_{m-2}} \\
& -\frac{u_{1} V_{13}^{(n)} y\left(y+A_{m} / V_{13}^{(n)}\right)-V_{12}^{(n)} y\left(y+\mathscr{A}_{m} / V_{12}^{(n)}\right)}{E_{m-2}} \\
& +\frac{2}{3} i \lambda \underset{\lambda \rightarrow \mu_{j}\left(x, t_{r}\right)}{=}-\frac{\mu_{j, x}\left(x, t_{r}\right)}{\lambda-\mu_{j}\left(x, t_{r}\right)}+O(1) \underset{\lambda \rightarrow \mu_{j}\left(x, t_{r}\right)}{=} \partial_{x} \ln \\
& \cdot\left(\lambda-\mu_{j}\left(x, t_{r}\right)\right)+O(1) \\
& \tilde{V}_{11}^{(r)}\left(\lambda, x, t_{r}\right)+\tilde{V}_{12}^{(r)}\left(\lambda, x, t_{r}\right) \phi_{2}\left(P, x, t_{r}\right)+\tilde{V}_{13}^{(r)}\left(\lambda, x, t_{r}\right) \phi_{3} \\
& \cdot\left(P, x, t_{r}\right)=\tilde{V}_{11}^{(r)}+\tilde{V}_{12}^{(r)} \frac{y^{2} V_{13}^{(n)}-y A_{m}+B_{m}}{E_{m-2}} \\
& -\tilde{V}_{13}^{(r)} \frac{y^{2} V_{12}^{(n)}-y \mathscr{A}_{m}+\mathscr{B}_{m}}{E_{m-2}} \\
& =\frac{1}{E_{m-2}}\left[\left(\tilde{V}_{12}^{(r)} V_{13}^{(n)}-\tilde{V}_{13}^{(r)} V_{12}^{(n)}\right) y^{2}-\left(\tilde{V}_{12}^{(r)} A_{m}-\tilde{V}_{13}^{(r)} \mathscr{A}_{m}\right) y\right. \\
& \left.+\frac{1}{3} E_{m-2, t_{r}}-\frac{2}{3}\left(\tilde{V}_{13}^{(r)} V_{12}^{(n)}-\tilde{V}_{12}^{(r)} V_{13}^{(n)}\right) S_{m}\right] \\
& =\frac{1}{3} \frac{E_{m-2, t_{r}}}{E_{m-2}}+\frac{2}{3} \frac{\left(\tilde{V}_{12}^{(r)} V_{13}^{(n)}-\tilde{V}_{13}^{(r)} V_{12}^{(n)}\right)\left(3 y^{2}+S_{m}\right)}{E_{m-2}} \\
& -\frac{\tilde{V}_{12}^{(r)} V_{13}^{(n)} y\left(y+A_{m} / V_{13}^{(n)}\right)-\tilde{V}_{13}^{(r)} V_{12}^{(n)} y\left(y+\mathscr{A}_{m} / V_{12}^{(n)}\right)}{E_{m-2}} \\
& \underset{\lambda \rightarrow \mu_{j}\left(x, t_{r}\right)}{=} \frac{\mu_{j,, t_{r}}\left(x, t_{r}\right)}{\lambda-\mu_{j}\left(x, t_{r}\right)}+O(1)_{\lambda \rightarrow \mu_{j}\left(x, t_{r}\right)}^{=} \partial_{t_{r}} \ln \\
& \cdot\left(\lambda-\mu_{j}\left(x, t_{r}\right)\right)+O(1) .
\end{aligned}
$$


Then,

$$
\begin{aligned}
& \psi_{1}\left(P, x, x_{0}, t_{r}, t_{0, r}\right)=\exp \left(\int _ { x _ { 0 } } ^ { x } \left[i \lambda+u_{1}\left(x^{\prime}, t_{r}\right) \phi_{2}\left(P, x^{\prime}, t_{r}\right)\right.\right. \\
& \left.+\phi_{3}\left(P, x^{\prime}, t_{r}\right)\right] d x^{\prime}+\int_{t_{0, r}}^{t_{r}}\left[\tilde{V}_{11}^{(r)}\left(\lambda, x_{0}, t^{\prime}\right)+\tilde{V}_{12}^{(r)}\left(\lambda, x_{0}, t^{\prime}\right)\right. \\
& \left.\left.\cdot \phi_{2}\left(P, x_{0}, t^{\prime}\right)+\tilde{V}_{13}^{(r)}\left(\lambda, x_{0}, t^{\prime}\right) \phi_{3}\left(P, x_{0}, t^{\prime}\right)\right] d t^{\prime}\right) \\
& =\frac{\lambda-\mu_{j}\left(x, t_{r}\right)}{\lambda-\mu_{j}\left(x_{0}, t_{r}\right)} \frac{\lambda-\mu_{j}\left(x_{0}, t_{r}\right)}{\lambda-\mu_{j}\left(x_{0}, t_{0, r}\right)} O(1)=\frac{\lambda-\mu_{j}\left(x, t_{r}\right)}{\lambda-\mu_{j}\left(x_{0}, t_{0, r}\right)} O(1) \\
& = \begin{cases}\left(\lambda-\mu_{j}\left(x, t_{r}\right)\right) O(1), & \text { for } P \text { near } \widehat{\mu}_{j}\left(x, t_{r}\right) \neq \widehat{\mu}_{j}\left(x_{0}, t_{0, r}\right), \\
O(1), & \text { for } P \text { near } \widehat{\mu}_{j}\left(x, t_{r}\right)=\widehat{\mu}_{j}\left(x_{0}, t_{0, r}\right), \\
\left(\lambda-\mu_{j}\left(x_{0}, t_{0, r}\right)\right)^{-1} O(1), & \text { for } P \text { near } \widehat{\mu}_{j}\left(x_{0}, t_{0, r}\right) \neq \widehat{\mu}_{j}\left(x, t_{r}\right),\end{cases}
\end{aligned}
$$

where $O(1) \neq 0$. Consequently, all zeros and poles of $\psi_{1}$ and $\Psi_{1}$ on $\mathscr{K}_{m-2} \backslash\left\{P_{\infty_{1}}, P_{\infty_{2}}, P_{\infty_{3}}\right\}$ are simple and coincident. Similar to Theorem 4, one can find that $\psi_{1}$ and $\Psi_{1}$ have the same essential singularities at $P_{\mathrm{O}_{1}}, P_{\mathrm{\infty}_{2}}, P_{\mathrm{\infty}_{3}}$. Then, the Riemann-Roch uniqueness results in $\Psi_{1}=\psi_{1}$.

We can explicitly rewrite $\theta\left(\underline{z}\left(P_{\infty_{j}}, \underline{\widehat{\mu}}\left(x, t_{r}\right)\right)\right), \theta\left(\underline{z}\left(P_{\infty_{j}}\right.\right.$, $\left.\left.\underline{\widehat{\hat{v}}}\left(x, t_{r}\right)\right)\right)$, and $\theta\left(\underline{z}\left(P_{\infty_{j}}, \underline{\hat{\xi}}\left(x, t_{r}\right)\right)\right)$ as

$$
\begin{aligned}
& \theta\left(\underline{z}\left(P_{\infty_{j}}, \underline{\mu}\left(x, t_{r}\right)\right)\right)=\theta\left(M_{1}^{(j)}+U_{2}^{(2)} x+\tilde{U}_{r+1}^{(2)} t_{r}\right), \\
& \theta\left(\underline{z}\left(P_{\infty_{j}}, \underline{v}\left(x, t_{r}\right)\right)\right)=\theta\left(M_{2}^{(j)}+U_{2}^{(2)} x+\tilde{U}_{r+1}^{(2)} t_{r}\right), \\
& \theta\left(\underline{z}\left(P_{\infty_{j}}, \underline{\xi}\left(x, t_{r}\right)\right)\right)=\theta\left(M_{3}^{(j)}+U_{2}^{(2)} x+\tilde{U}_{r+1}^{(2)} t_{r}\right),
\end{aligned}
$$

where-

$M_{s}^{(j)}=M-\mathscr{A}\left(P_{\infty_{j}}\right)+\underline{\rho}^{(s)}\left(x_{0}, t_{0, r}\right)-U_{2}^{(2)} x_{0}-\tilde{U}_{r+1}^{(2)} t_{0, r}, j=1,2$ , 3, $s=1,2,3$, with the aid of the following theorem.

Theorem 10. Assume that the curve $\mathscr{K}_{m-2}$ is nonsingular and let $\left(x, t_{r}\right),\left(x_{0}, t_{0, r}\right) \in \mathbb{C}^{2}$. Then,

$$
\begin{aligned}
& \rho^{(1)}\left(x, t_{r}\right)=\rho^{(1)}\left(x_{0}, t_{0, r}\right)+U_{2}^{(2)}\left(x-x_{0}\right)+\tilde{U}_{r+1}^{(2)}\left(t_{r}-t_{0, r}\right), \\
& \rho^{(2)}\left(x, t_{r}\right)=\rho^{(2)}\left(x_{0}, t_{0, r}\right)+U_{2}^{(2)}\left(x-x_{0}\right)+\tilde{U}_{r+1}^{(2)}\left(t_{r}-t_{0, r}\right), \\
& \rho^{(3)}\left(x, t_{r}\right)=\rho^{(3)}\left(x_{0}, t_{0, r}\right)+U_{2}^{(2)}\left(x-x_{0}\right)+\tilde{U}_{r+1}^{(2)}\left(t_{r}-t_{0, r}\right) .
\end{aligned}
$$

Proof. First, we consider a meromorphic differential

$$
\Omega\left(x, x_{0}, t_{r}, t_{0, r}\right)=\frac{\partial}{\partial \lambda} \ln \left(\psi_{1}\left(P, x, x_{0}, t_{r}, t_{0, r}\right)\right) d \lambda .
$$

From the representation (180), we have

$$
\begin{aligned}
& \Omega\left(x, x_{0}, t_{r}, t_{0, r}\right)=-\left(x-x_{0}\right) \Omega^{(2)}-\left(t_{r}-t_{0, r}\right) \tilde{\Omega}_{r+1}^{(2)} \\
& -\sum_{j=1}^{m-2} \omega_{\widehat{\mu}\left(x_{0}, t_{0, r}\right), \widehat{\mu}\left(x, t_{r}\right)}^{(3)}+\tilde{\omega},
\end{aligned}
$$

where $\tilde{\omega}$ denotes a holomorphic differential on $\mathscr{K}_{m-2}$, that is, $\tilde{\omega}=\sum_{j=1}^{m-2} \omega_{j} e_{j}$ for some $e_{j} \in \mathbb{C}, j=1, \cdots, m-2$. Because $\psi_{1}\left(P, x, x_{0}, t_{r}, t_{0, r}\right)$ is single-valued on $\mathscr{K}_{m-2}$, all $a$ - and $b$ -periods of $\Omega$ are integer multiples of $2 \pi i$, and hence,

$$
2 \pi i m_{k}=\int_{a_{k}} \Omega\left(x, x_{0}, t_{r}, t_{0, r}\right)=\int_{a_{k}} \tilde{\omega}=e_{k}, \quad k=1, \cdots, m-2,
$$

for some $m_{k} \in \mathbb{Z}$. Similarly, for some $n_{k} \in \mathbb{Z}$,

$$
\begin{aligned}
2 \pi i n_{k}= & \int_{b_{k}} \Omega\left(x, x_{0}, t_{r}, t_{0, r}\right)=-\left(x-x_{0}\right) \int_{b_{k}} \Omega^{(2)} \\
& -\left(t_{r}-t_{0, r}\right) \int_{b_{k}} \tilde{\Omega}_{r+1}^{(2)}+\sum_{j=1}^{m-2} \int_{b_{k}} \omega_{\widehat{\mu}_{j}\left(x, t_{r}\right)}^{(3)} \widehat{\mu}_{j}\left(x_{0}, t_{0, r}\right) \\
= & \int_{b_{k}} \tilde{\omega}-\left(x-x_{0}\right) \int_{b_{k}} \Omega^{(2)}-\left(t_{r}-t_{0, r}\right) \int_{b_{k}} \tilde{\Omega}_{r+1}^{(2)}+2 \pi i \\
& \cdot \sum_{j=1}^{m-2} \int_{\widehat{\mu}_{j}\left(x_{0}, t_{0, r}\right)}^{\mu_{j}\left(x, t_{r}\right)} \omega_{k}=2 \pi i \sum_{j=1}^{m-2} m_{j} \int_{b_{k}} \omega_{j}-2 \pi i\left(x-x_{0}\right) U_{2, k}^{(2)} \\
& -2 \pi i\left(t_{r}-t_{0, r}\right) \tilde{U}_{r+1, k}^{(2)}+2 \pi i \sum_{j=1}^{m-2} m_{j} \tau_{j, k} \\
& +2 \pi i\left[\sum_{j=1}^{m-2} \int_{Q_{0}}^{\mu_{j}\left(x, t_{r}\right)} \omega_{k}-\sum_{j=1}^{m-2} \int_{Q_{0}}^{\mu_{j}\left(x_{0}, t_{0, r}\right)} \omega_{k}\right],
\end{aligned}
$$

in terms of

$$
\int_{b_{k}} \omega_{Q_{1}, Q_{2}}^{(3)}=2 \pi i \int_{Q_{1}}^{Q_{2}} \omega_{k}, \quad k=1, \cdots, m-1
$$

By symmetry of $\tau$, this is equivalent to $\left(\left(x, t_{r}\right) \in \Omega_{\mu}\right)$

$$
\rho^{(1)}\left(x, t_{r}\right)=\rho^{(1)}\left(x_{0}, t_{0, r}\right)+U_{2}^{(2)}\left(x-x_{0}\right)+\tilde{U}_{r+1}^{(2)}\left(t_{r}-t_{0, r}\right) .
$$

Using this equality and the linear equivalence $\mathfrak{D}_{P_{\infty_{1}}, \underline{\widehat{\mu}}\left(x, t_{r}\right)} \sim \mathfrak{D}_{P_{\infty_{2}}, \underline{\widehat{v}}\left(x, t_{r}\right)}$, and $\mathfrak{D}_{P_{\infty_{1}}, \underline{\widehat{\mu}}\left(x, t_{r}\right)} \sim \mathfrak{D}_{P_{\infty_{3}}, \underline{\underline{\xi}}\left(x, t_{r}\right)}$, that is,

$$
\begin{aligned}
& \mathscr{A}\left(P_{\mathrm{O}_{1}}\right)+\rho^{(1)}\left(x, t_{r}\right)=\mathscr{A}\left(P_{\mathrm{O}_{2}}\right)+\rho^{(2)}\left(x, t_{r}\right), \\
& \mathscr{A}\left(P_{\mathrm{O}_{1}}\right)+\rho^{(1)}\left(x, t_{r}\right)=\mathscr{A}\left(P_{\mathrm{O}_{3}}\right)+\rho^{(3)}\left(x, t_{r}\right),
\end{aligned}
$$


we can present the other two equalities

$$
\begin{aligned}
& \rho^{(2)}\left(x, t_{r}\right)=\rho^{(2)}\left(x_{0}, t_{0, r}\right)+U_{2}^{(2)}\left(x-x_{0}\right)+\tilde{U}_{r+1}^{(2)}\left(t_{r}-t_{0, r}\right), \\
& \rho^{(3)}\left(x, t_{r}\right)=\rho^{(3)}\left(x_{0}, t_{0, r}\right)+U_{2}^{(2)}\left(x-x_{0}\right)+\tilde{U}_{r+1}^{(2)}\left(t_{r}-t_{0, r}\right) .
\end{aligned}
$$

\section{Conclusions}

In this paper, firstly we obtain the CCIRD hierarchy related to a $3 \times 3$ matrix spectral problem based on the Lenard recursion equations, and a trigonal curve $\mathscr{K}_{m-2}$ of arithmetic genus $m-2$ with three infinite points is introduced by using the characteristic polynomial of Lax matrix for the stationary CCIRD equations, from which the stationary Baker-Akhiezer function and associated meromorphic functions are given on $\mathscr{K}_{m-2}$. Then, the stationary CCIRD equations are decomposed into the system of Dubrovin-type ordinary differential equations. Furthermore, we present the explicit theta function representations of the stationary Baker-Akhiezer function, of the meromorphic functions, and, in particular, of the potentials for the entire stationary CCIRD hierarchy. Finally, we extend all the Baker-Akhiezer function, the meromorphic functions, the Dubrovin-type equations, and the theta function representations dealt with in Sections 3 and 4 to the time-dependent case. The technology presented in this paper can be applied to other hierarchies related to $3 \times$ 3 matrix spectral problems, to get more various algebrogeometric solutions, which will enrich and supplement the known results.

\section{Data Availability}

No data were used to support this study.

\section{Conflicts of Interest}

The authors declare that they have no conflicts of interest.

\section{Acknowledgments}

This work was supported by the Natural Science Foundation of China (Grant Nos. 11971475 and 11805114), the Natural Science Foundation of Shandong Province (Grant Nos. ZR2016AL04, ZR2016FL05, and ZR2017MF039), and the High-Level Training Project of Taishan Medical University (No. 2015GCC07).

\section{References}

[1] A. Yusuf, F. Tchier, and M. Inc, "New interaction and combined multi-wave solutions for the Heisenberg ferromagnetic spin chain equation," The European Physical Journal Plus, vol. 135 , no. $5,2020$.

[2] T. A. Sulaiman, A. Yusuf, and A. Atangana, "New lump, lumpkink, breather waves and other interaction solutions to the (3 +1 )-dimensional soliton equation," Communications in Theoretical Physics, vol. 72, no. 8, article 085004, 2020.
[3] F. Tchier, M. Inc, and A. Yusuf, "Symmetry analysis, exact solutions and numerical approximations for the space-time Carleman equation in nonlinear dynamical systems," The European Physical Journal Plus, vol. 134, no. 6, p. 250, 2019.

[4] M. L. Wang and X. Bai, "Exact solutions for a generalized Burgers-Fisher equation," Journal of Lanzhou University(Natural Sciences), vol. 35, no. 2, pp. 1-6, 1999.

[5] E. D. Belokolos, A. I. Bobenko, V. Z. Enol'skii, A. R. Its, and V. B. Matveev, Algebro-Geometric Approach to Nonlinear Integrable Equations, Springer, Berlin, 1994.

[6] V. Batchenko and F. Gesztesy, "On the spectrum of Schrödinger operators with quasi-periodic algebro-geometric KDV potentials," Journal d'Analyse Mathématique, vol. 95, no. 1, pp. 333-387, 2005.

[7] P. D. Lax, "Periodic solutions of the KdV equation," Communications on Pure and Applied Mathematics, vol. 28, no. 1, pp. 141-188, 1975.

[8] E. Date and S. Tanaka, "Periodic multi-soliton solutions of Korteweg-de Vries equation and Toda lattice," Progress of Theoretical Physics Supplement, vol. 59, pp. 107-125, 1976.

[9] Y. C. Ma and M. J. Ablowitz, "The periodic cubic Schrödinger equation," Applications of Mathematics, vol. 65, no. 2, pp. 113$158,1981$.

[10] C. Yue and T. Xia, "Algebro-geometric solutions for the complex Sharma-Tasso-Olver hierarchy," Journal of Mathematical Physics, vol. 55, no. 8, pp. 083511-083535, 2014.

[11] Z. Qiao, " $r$-matrix and algebraic-geometric solution for integrable symplectic map," Chinese Science Bulletin, vol. 44, no. 2, pp. 114-118, 1999.

[12] Z. Qiao, "Generalized $r$-matrix structure and algebrogeometric solution for integrable system," Reviews in Mathematical Physics, vol. 13, no. 5, pp. 545-586, 2001.

[13] R. Zhou, "Dynamical $r$-matrices for the constrained HarryDym flows," Physics Letters A, vol. 220, no. 6, pp. 320-330, 1996.

[14] R. Zhou, "The finite-band solution of the Jaulent-Miodek equation," Journal of Mathematical Physics, vol. 38, no. 5, pp. 2535-2546, 1997.

[15] H. Pan, T. Xia, and D. Chen, "A two-component generalization of Burgers' equation with quasi-periodic solution," Reports on Mathematical Physics, vol. 74, no. 2, pp. 235-250, 2014.

[16] I. M. Krichever, "Algebraic-geometric construction of the Zaharov-Sabat equations and their periodic solutions," Doklady Akademii Nauk SSSR, vol. 227, pp. 394-397, 1976.

[17] I. M. Krichever, "Integration of nonlinear equations by the methods of algebraic geometry," Functional Analysis and Its Applications, vol. 11, no. 1, pp. 12-26, 1977.

[18] B. A. Dubrovin, "Completely integrable Hamiltonian systems associated with matrix operators and Abelian varieties," Functional Analysis and Its Applications, vol. 11, no. 4, pp. 265-277, 1978.

[19] B. A. Dubrovin, "Theta functions and nonlinear equations," Russian Mathematical Surveys, vol. 36, no. 2, pp. 11-92, 1981.

[20] B. A. Dubrovin, "Matrix finite-gap operators," Revue Scientifique et Technique, vol. 23, pp. 33-78, 1983.

[21] F. Gesztesy and R. Ratnaseelan, "An alternative approach to algebro-geometric solutions of the AKNS hierarchy," Reviews in Mathematical Physics, vol. 10, no. 3, pp. 345-391, 1998.

[22] F. Gesztesy and H. Holden, "Algebro-geometric solutions of the Camassa-Holm hierarchy," Revista Matemática Iberoamericana, vol. 19, pp. 73-142, 2003. 
[23] F. Gesztesy and H. Holden, Soliton Equations and Their Algebro-Geometric Solutions. Vol. I:(1+1)- Dimensional Continuous Models, vol. 79 of Cambridge Studies in Advanced Mathematics, Cambridge University Press, Cambridge, 2003.

[24] F. Gesztesy, H. Holden, J. Michor, and G. Teschl, Soliton Equations and Their Algebro-Geometric Solutions, Vol. II: (1+1)Dimensional Discrete Models, vol. 114 of Cambridge Studies in Advanced Mathematics, Cambridge University Press, Cambridge, 2008.

[25] J. S. Geronimo, F. Gesztesy, and H. Holden, “Algebro-geometric solutions of the Baxter-Szegő difference equation," Communications in Mathematical Physics, vol. 258, no. 1, pp. 149-177, 2005.

[26] W. Bulla, F. Gesztesy, H. Holden, and G. Teschl, "Algebro-geometric quasi-periodic finite gap solutions of the Toda and Kacvan Moerbeke hierarchies," Amer. Math. Soc., vol. 135, pp. 179, 1998.

[27] E. Fan, "The positive and negative Camassa-Holm- $\gamma$ hierarchies, zero curvature representations, bi-Hamiltonian structures, and algebro-geometric solutions," Journal of Mathematical Physics, vol. 50, no. 1, pp. 013525-013547, 2009.

[28] R. Dickson, F. Gesztesy, and K. Unterkofler, "Algebro-geometric solutions of the Boussinesq hierarchy," Reviews in Mathematical Physics, vol. 11, no. 7, pp. 823-879, 1999.

[29] G. He, X. Geng, and L. Wu, "Algebro-geometric quasi-periodic solutions to the three-wave resonant interaction hierarchy," SIAM Journal on Mathematical Analysis, vol. 46, no. 2, pp. 1348-1384, 2014.

[30] H. Wang and X. G. Geng, "Algebro-geometric solutions to a new hierarchy of soliton equations," Journal of Mathematical Physics, Analysis, Geometry, vol. 11, no. 4, pp. 359-398, 2015.

[31] W.X. Ma, "Trigonal curves and algebro-geometric solutions to soliton hierarchies I," Proceedings of the Royal Society A: Mathematical, Physical and Engineering Sciences, vol. 473, no. 2203, p. 20170232, 2017.

[32] W. X. Ma, "Trigonal curves and algebro-geometric solutions to soliton hierarchies II," Proceedings of the Royal Society A: Mathematical, Physical and Engineering Sciences, vol. 473, no. 2203, p. 20170233, 2017.

[33] Y. Hou, P. Zhao, E. Fan, and Z. Qiao, "Algebro-geometric solutions for the Degasperis-Procesi hierarchy," SIAM Journal on Mathematical Analysis, vol. 45, no. 3, pp. 1216-1266, 2013.

[34] Y. Hou, E. Fan, Z. Qiao, and Z. Wang, "Algebro-geometric solutions for the derivative Burgers hierarchy," Journal of Nonlinear Science, vol. 25, no. 1, pp. 1-35, 2015. 